\title{
Extreme Value Analysis of Intracellular Cargo Transport by Motor Proteins
}

\author{
Takuma Naoi ${ }^{1}$, Yuki Kagawa ${ }^{1}$, Kimiko Nagino ${ }^{1}$, Shinsuke Niwa ${ }^{2}$, and Kumiko Hayashi ${ }^{1,3 *}$ \\ ${ }^{1}$ Department of Applied Physics, Graduate School of Engineering, Tohoku University, Sendai, Japan \\ ${ }^{2}$ Frontier Research Institute for Interdisciplinary Sciences and Graduate School of Life Science, Tohoku \\ University, Sendai, Japan \\ ${ }^{3}$ Precursory Research for Embryonic Science and Technology (PRESTO), Japan Science and Technology \\ Agency (JST), Tokyo, Japan \\ *Correspondence to Kumiko Hayashi \\ kumiko@camp.apph.tohoku.ac.jp (K.H.)
}

\begin{abstract}
In the long axon of a neuron, cargo transport between the cell body and terminal synaptic region are mainly supported by the motor proteins kinesin and dynein, which are nano-sized drivers. Synaptic materials packed as cargos are anterogradely transported to the synaptic region by kinesin, whereas materials accumulated at the axon terminals are returned to the cell body by dynein. Extreme value analysis, typically used for disaster prevention in our society, was applied to analyze the velocity of kinesin and dynein nanosized drivers to disclose their physical properties in living cells.
\end{abstract}


Extreme value analysis (EVA) $[1,2]$ is a method developed to study a variety of topics in our society, such as disaster prevention [3], finance [4], sports [5], and human lifespan [6]. In this study, we extend the usage of EVA to investigate the nanoscale phenomena associated with the action of motor proteins inside cells. Motor protein is a general term for proteins that move and function using energy obtained from adenosine triphosphate (ATP) hydrolysis. They are elaborate nanosized molecular machines that work in our bodies. While myosin swings its lever arm to cause muscle contraction $[7,8]$, kinesin and dynein walk along microtubules to transport intracellular materials $[9,10]$, and $\mathrm{F}_{0} \mathrm{~F}_{1}$ synthase rotates to synthesize ATP molecules [11]. The physical properties of motor proteins, such as force and velocity, have been investigated mainly by in vitro singlemolecule experiments, in which the function of motor proteins consisting of minimal complexes were analyzed in glass chambers [12-17]. However, motor proteins function fully when they are in the intracellular environment and equipped with accessory proteins. Thus, the investigation of motor proteins in vivo is significant, although it is difficult to obtain information on the physical properties of their motion in the complex intracellular environment. EVA is a useful tool for investigating this, in particular their velocity, in cells because an extreme value at which the effect of environment is minimally affected is considered to be dependent mainly on the ability of motor proteins.

In the present study, we focused on the in vivo motion of synaptic cargo transport performed by motor proteins kinesin (UNC-104 [18,19]) and the cytoplasmic dynein [20], in the axons of motor neurons of living Caenorhabditis elegans (C. elegans) worms, a model organism in neuroscience. Synaptic materials packed as cargos are delivered to the synaptic region of the neuron by anterograde transport by kinesin, and unnecessary materials accumulated in the synaptic region are returned to the cell body by retrograde transport performed by dynein (Fig. 1(a)). Since the worm's bodies are transparent and their body movement is suppressed by anesthesia, the motion of fluorescently-labelled synaptic cargos in the living worms was observed by fluorescent microscopy (Fig. 1(b)), using a method described before [21]. The images were captured using a $150 \times$ 
objective lens and an sCMOS camera at 10 frames per second (see [22] for the detailed materials and methods). The kymograph analysis was performed using 'multi kymograph' in ImageJ [23] on all the recorded images to measure transport velocities (Fig. 1(b)). Velocity values were calculated as the slopes of the trajectories of the fluorescently-labelled cargos in the kymograph images.

Typically, a cargo exhibits moving motion at a constant velocity and pauses, rarely reversing its direction. The number of cargos exhibiting these movements is shown in Fig. 1(c). Our results confirmed that the directional reversal is a rare event, indicating few tug-of-war events, as is supported by the "motor coordination model," in which adaptor proteins that connect motors with a cargo activate or deactivate opposing motors [20]. Here, the tug-of-war is a phenomenon in which a cargo changes its direction by force comparison between the two opposing motors (kinesin and dynein) [24]. It is important to note that even if a load from the opposing motor is acting on a cargo, the application of EVA is valid (the tug-of-war effect on the EVA analysis was discussed in [22]) because a large viscous load, which is another opposing force, acts on the moving cargo in the first place. The viscous load values corresponding to various sizes of synaptic cargos (Fig. S1 of [22]) affect the velocity values as opposing loads in a high-viscosity intracellular environment of the cell. Indeed, the velocity distributions are broad (Fig. S2 of [22]), affected by the various load values acting on the cargo from the complex environment, and the physical properties of motor proteins seem to be difficult to ascertain from these velocity values. Under the circumstances, EVA was considered to highlight the difference between the motor types because it can evaluate velocity values at a condition similar to the no-load condition. Cargo size differences and the existence of opposing motors are demerits of physical measurement in complex intracellular environments, unlike the controlled situation of single-molecule experiment cases; however, in the present study, the unique difficulties observed in vivo were overcome through the use of EVA.

The velocity values of moving cargo during constant velocity segments were collected from a total of 232 worms. Approximately 5-20 velocity values were observed for each worm, from which the largest value $\left(v_{\max }^{i}\right)$ 
was chosen. Using $\left\{v_{\max }^{i}\right\}(i=1, \cdots, N$ where $N=228$ for anterograde transport and $N=217$ for retrograde transport), the return level plot, which is the typical analysis of EVA, defined as $\left(-1 / \log (i /(N+1)), \hat{v}_{\max }^{i}\right)$, was investigated (Figs. 2(a) and 2(b)), where $\left\{\hat{v}_{\max }^{i}\right\}$ is the rearranged data of $\left\{v_{\max }^{i}\right\}$ such that $\hat{v}_{\max }^{1} \leq$ $\hat{v}_{\max }^{2} \leq \cdots \leq \hat{v}_{\max }^{N}$. The two axis of the return level plot is called return period and return level, respectively. The return level plot indicates that the measured value (return level) occurs for the first time in the time period (return period). The strong point of the plot is that the values close to the rarely occurring extreme value can be enhanced due to the logarithmic expression of the $x$-axis. Because the return level plot for anterograde transport shows typical concave-up behavior (Fig. 2(a)), a property belonging to a Weibull distribution, then $V_{\max }$ was proved to exist and was estimated to be $3.9 \pm 0.4 \mu \mathrm{m} / \mathrm{s}$ by the equation given below.

$$
V_{\max }=\mu-\sigma / \xi
$$

where $\xi, \mu$, and $\sigma$ are the parameters of a Weibull distribution

$$
W\left(v_{\max }\right)=\exp \left[-\left\{1+\xi\left(\frac{v_{\max }-\mu}{\sigma}\right)\right\}^{-1 / \xi}\right] .
$$

The error of $V_{\max }$ consisted of fitting errors of $\xi, \mu$, and $\sigma$ (see [22] for the calculation), and $W\left(v_{\max }\right)$ obtained from the experimental data was fitted using the 'ismev' and 'evd' packages in R [25]. Note that the return level plot within the black dotted lines (Fig. 2(a)) representing the reliable section indicates the validity of the analysis. On the other hand, the return level plot of retrograde transport (Fig. 2(b)) shows a peculiar behavior showing two-step convergences. When the retrograde data was separated by the change point $\left(i=N_{\mathrm{c}}\right)$ depicted in Fig. 2(b) (black arrow) as $\left\{v_{\max }^{i}\right\}\left(i=1, \cdots, N_{\mathrm{c}}\right)$ and $\left\{v_{\max }^{i}\right\}\left(i=N_{\mathrm{c}}+1, \cdots, N\right)$, each return level plot showed the behavior of a Weibull distribution. Note that the changing point was determined as the intersection of the fitting lines (dotted lines in Fig. 2(b)). Then, by using Eq. (1), $V_{\max }$ could be estimated for each to be $4.1 \pm 0.4 \mu \mathrm{m} / \mathrm{s}$ or $6.5 \pm 1.0 \mu \mathrm{m} / \mathrm{s}$, respectively. In Figs. 2(c) and 2(d), the probability distributions $w\left(v_{\max }\right)\left(=W\left(v_{\max }\right) / \mathrm{d} v_{\max }\right)$ for anterograde and retrograde transport are shown. In the case of retrograde transport, $w_{1}\left(v_{\max }\right)$ and $w_{2}\left(v_{\max }\right)$ are the fitting functions of the probability distributions for $\left\{v_{\max }^{i}\right\}(i=$ 
$\left.1, \cdots, N_{\mathrm{c}}\right)$ and $\left\{v_{\max }^{i}\right\}\left(i=N_{\mathrm{c}}+1, \cdots, N\right)$, while $w_{2}\left(v_{\max }\right)$ does not exist for the anterograde case. Note that the block sizes (representing the number of worms) from which $v_{\max }^{i}$ was chosen (Fig. S4 of [22]) and bootstrapping analysis of the data $\left\{v_{\text {max }}^{i}\right\}$ (Fig. S3 of [22]) were investigated to validate the results.

We consider that the two-step convergence behavior was only observed for retrograde transport by dynein (Fig. 2(b)) from the viewpoint of the force-velocity relationship of motor proteins, representing the load dependence of velocity. According to the results of previous studies using single-molecule experiments, it is known that two regimes exist in the force-velocity curves of motor proteins: load-sensitive and load-insensitive regimes [26] (Figs. 3(a) and 3(b)). In one, the velocity changes rapidly with the increase in load $(F)$, while in the other the velocity changes only slightly with the increase in load. In vitro single-molecule experiments revealed that the force-velocity curve of kinesin was concave-down (Fig. 3(a)) [12], while that of dynein (except yeast dynein $[13,14]$ ) was concave-up (Fig. 3(b)) $[15,16,27]$. This mechanical difference can be explained as follows: kinesin keeps moving $8 \mathrm{~nm}$ (the interval of the microtubule structural unit) along a microtubule per single ATP hydrolysis even when a low load is applied, which makes its force-velocity relation load-insensitive and leads to a concave-down force-velocity curve. On the other hand, dynein, which can take variable step sizes of $8-40 \mathrm{~nm}$ under no load $[13,15,17,27]$, slows down rapidly by decreasing the step size even when a low load is applied, leading to a rapid velocity decrease and a concave-up force-velocity curve. Because the simplest model of the force-velocity curve can be characterized by the changing point $\left(F_{\mathrm{c}}, v_{\mathrm{c}}\right)$ (Figs. 3(a) and 3(b)) between the load-sensitive and load-insensitive regimes, in the following sections, we aim to estimate the most probable values $\left(F_{\mathrm{c}}^{*}\right.$ and $v_{\mathrm{c}}^{*}$ ), which reproduces the return level plots shown in Figs. 2(a) and 2(b), by performing numerical simulations. Note that in the following simulation, the axes of the force-velocity relationship are normalized as $\left(F / F_{\mathrm{S}}, v / v(F=0)\right)$, where $F_{\mathrm{S}}$ is the stall force of a motor protein [12-17], which is the maximum force the motor can generate against an opposing load. Since in the present study the absolute values of in vivo force could not be measured in the living worms, we investigated only the outlines of 
the force-velocity relationships.

A simulated value of the transport velocity is generated stochastically by using the force-velocity curve (Fig. 3), where various cargo sizes make the velocity value stochastic. The probability distribution of the cargo size can be estimated from the distribution of the fluorescence intensity (FI) of the cargo (Fig. S1 of [22]) through the relation FI $\propto 4 \pi r^{2}$ ( $r$ : radius of a cargo), assuming that fluorescence proteins labelling a cargo are uniformly distributed on its surface. Then, a slope $\alpha$ of the straight line $v=\alpha F$ representing the Stokes' law is generated based on the equation $\alpha=c / r$, where $c$ is a constant and $1 / r$ is stochastically generated based on the Gamma distribution of $1 / \sqrt{\mathrm{FI}}$ (Fig. S1 of [22]). A simulated velocity value $v_{\text {sim }}$ can be obtained as the intersection between the line and the force velocity curve. Here, the proportional constant $c$ is fixed so that the distribution of the simulated velocity $\left(v_{\text {sim }}\right)$ matches that of the experimentally measured velocity $(v)$ (Fig. S2 of [22]). The gray lines depicted in Fig. 3 represent $95 \%$ confidence interval of $\alpha$. The $v_{\text {sim,max }}^{i}$ was chosen among the 10 values of $v_{\text {sim }}$. This procedure was repeated 200 times (i.e., $i=1, \cdots, N$ where $N=200$ ). The probability distributions $w_{1}^{\text {sim }}$ and $w_{2}^{\text {sim }}$ were calculated from the dataset $\left\{v_{\text {sim,max }}^{i}\right\}$, although $w_{2}^{\text {sim }}$ did not exist for anterograde transport. Here, the extreme value dataset $\left\{\hat{v}_{\text {sim,max }}^{i}\right\}$ was the rearranged data of $\left\{v_{\text {sim,max }}^{i}\right\}$ such that $\hat{v}_{\text {sim,max }}^{1} \leq \hat{v}_{\text {sim,max }}^{2} \leq \cdots \leq \hat{v}_{\text {sim,max }}^{N}$

Comparing the extreme value distributions $w_{1}^{\text {sim }}$ and $w_{2}^{\text {sim }}$ with $w_{1}$ and $w_{2}$ (Figs. 2(c) and 2(d)), the error function, $E\left(F_{\mathrm{c}}, v_{\mathrm{c}}\right)$, is defined as:

$$
\begin{aligned}
E\left(F_{\mathrm{c}}, v_{\mathrm{c}}\right)= & \frac{1}{N_{\mathrm{c}}} \sum_{i=1}^{N_{\mathrm{c}}}\left\{w_{1}\left(\hat{v}_{\text {sim, max }}^{i}\right)-w_{1}^{\mathrm{sim}}\left(\hat{v}_{\text {sim, max }}^{i}\right)\right\}^{2} \\
& +\frac{1}{N-N_{\mathrm{c}}} \sum_{i=N_{\mathrm{c}}+1}^{N}\left\{w_{2}\left(\hat{v}_{\text {sim, } \max }^{i}\right)-w_{2}^{\operatorname{sim}}\left(\hat{v}_{\text {sim, max }}^{i}\right)\right\}^{2},
\end{aligned}
$$

where $w_{2}^{\text {sim }}$ does not exist and $N_{\mathrm{c}}=1$ for anterograde transport. For each $\left(F_{\mathrm{c}}, v_{\mathrm{c}}\right)$, the dataset $\left\{\hat{v}_{\text {sim,max }}^{i}\right\}$ was generated numerically and repeatedly ten times, and $E\left(F_{c}, v_{c}\right)$ was plotted as the mean of the 10 trials (Figs. 4(a) and 4(b)). The $E\left(F_{\mathrm{c}}, v_{\mathrm{c}}\right)$ was calculated using the steepest descent method ('SciPy' package in 
Python). In the anterograde case, the red region exists above the diagonal line (Fig. 4(a)), and below the diagonal line in the retrograde case exits (Fig. 4(b)). These results indicate that the force-velocity relationship for the anterograde case was concave-down and that for the retrograde case was concave-up.

The values of $F_{\mathrm{c}}^{*}$ (or $v_{\mathrm{c}}^{*}$ ) were defined as the mean of $\left\{F_{\mathrm{c}}\right\}$ (or $\left\{v_{\mathrm{c}}\right\}$ ) belonging to the top $10 \%$ minimum values of $E\left(F_{\mathrm{c}}, v_{\mathrm{c}}\right) .\left(F_{\mathrm{c}}^{*}, v_{\mathrm{c}}^{*}\right)=(0.61 \pm 0.20(\mathrm{SE}), 0.96 \pm 0.05(\mathrm{SE}))$ for anterograde transport and by $\left(F_{\mathrm{c}}^{*}, v_{\mathrm{c}}^{*}\right)=(0.63 \pm 0.1(\mathrm{SE}), 0.25 \pm 0.0(\mathrm{SE}))$ for retrograde transport. With $\left(F_{\mathrm{c}}^{*}, v_{\mathrm{c}}^{*}\right)$, the return level plots of $\left\{\hat{v}_{\text {sim,max }}^{i}\right\}$ for both anterograde and retrograde transport were reproduced by numerical simulation (Figs. 4(c) and 4(d)). As shown in Fig. 4(f), the peculiar part (violet symbols) in Fig. 4(d) for retrograde transport corresponds to the velocity values in the load-sensitive regime in Fig. 4(f). The steep velocity decrease in the low-load condition for the retrograde case caused a major variation in the larger values of $v_{\text {sim,max }}^{i}$, while the velocity values in the load-insensitive regime resulted in a minor variation in the large values of $v_{\text {sim,max }}^{i}$ (Fig. 4(e)), and the return level plot showed the Weibull type convergence in the anterograde case (Fig. 4(c)). Thus, the outline of in vivo force-velocity relations was estimated using the experimental results of EVA.

In summary, in this paper, we applied EVA for the first time to cargo transport by motor proteins kinesin and dynein in the neurons of living worms, which was observed by high resolution fluorescence microscopy. We investigated the velocity values of the transport, and found that the return level plots of the extreme values of velocity disclosed the difference between the motor types. Using the simulation, the difference was attributed to the fact that the force-velocity relationship for the anterograde transport was concave-down and that for the retrograde case was concave-up. Although the physical difference was not clearly shown in the general velocity distributions (Fig. S2 of [22]) because a high-viscosity intracellular environment affected them, EVA succeeded in highlighting the difference by evaluating velocity values at a condition similar to the no-load condition. In the following, we discuss several issues related to the main results.

It has been suggested that a single cargo is transported by multiple motors. The effect of multiple motor 
transport on the results of the present study was investigated (Fig. S5 of [22]). Previously, we used a noninvasive force measurement technique, developed by our research group, on the neurons of C. elegans [28-30] and estimated that the number of motors carrying a synaptic cargo was 1-3 [21]. While considering the effect of multiple motor proteins in the modification of the force-velocity curves, we found a concave-down/concaveup force-velocity relationship for anterograde/retrograde transport.

We then referred to the chemo-mechanical coupling models of the force-velocity relationship of kinesin and dynein theoretically derived based on the ATP hydrolysis mechanisms of motor proteins. Force-velocity relationships are derived from the one-state [15] and three-state models [31] of motor proteins, as well as an energy-landscape model [12]. Regarding the return level plot for these chemo-mechanical coupling models (Fig. S8-S11), we found similar tendencies to those observed using the simple force-velocity relationships (Fig. 3). The results are summarized in [22].

Recent results of in vitro single-molecule experiments suggested a concave-up force-velocity relationship for ciliary [16] and mammalian dynein [15,27], while yeast dynein exhibits a concave-down (kinesin-like) force-velocity relationship $[13,14]$. In the present study, we found a concave-up force-velocity relationship for the cytoplasmic dynein in C. elegans. For the investigation of mammalian dynein, EVA was also applied to the data of synaptic cargo transport in mouse hippocampal neurons originally measured in Ref. [28]. Two-step convergence in the return level plot was also observed for retrograde transport (Fig. S12 of [22]), which corresponds to the concave-up force velocity relationship reported in previous studies [15,27]. Interestingly, several dynein motors showed a concave-up force velocity curve, biological significance of which regarding collective cargo transport by multiple motor proteins is explained below, and was first introduced in a previous study [27]. When multiple motors work together, the leading dynein decreases its velocity rapidly in the presence of low load so that the trailing dynein can catch up. This allows the trailing dynein to share the load with the leading dynein, preventing detachment of the leading dynein from the microtubules. In other words, 
the rapid decrease in velocity in the load-sensitive region results in self-correction of the dynein position, allowing them to move as a loosely bunched group [27]. On the other hand, leading kinesin does not slow down according to a concave-down force-velocity relationship in the presence of low load. As a result, trailing kinesin cannot catch up to the leading kinesin, causing it to easily detach from microtubules [27].

Finally, another peculiar behavior of the return level plot for synaptic cargo transport is that of pathogenic mutant kinesin, which causes hereditary spastic paralysis and force and velocity impairment [32,33], according to our previous study [32]. Interpretation of the return level plot based on the force-velocity relationship is a promising tool for future research on neuronal diseases, particularly KIF1A-associated neurological disorder [34,35] KIF1A is a type of kinesin transporting synaptic vesicle precursor cargo. Because in vivo force measurement is difficult, estimation of physical properties using EVA can be helpful for understanding the in vivo behavior of motor proteins. We believe that the findings of the present study represent a step forward in broadening the scope of EVA applications.

\section{ACKNOWLEDGMENTS}

We acknowledge Dr. T. Saigo for discussions on EVA, and Dr. K. Sasaki for discussions on motor proteins. We would like to thank Editage (www.editage.com) for English language editing. This work was supported by JST PRESTO (grant no. JPMJPR1877) and the FRIS Creative Interdisciplinary Research Program, Tohoku University to K. H., and by JSPS KAKENHI (grant Nos. 19H04738, 20H03247, and 20K21378) to S. N. 

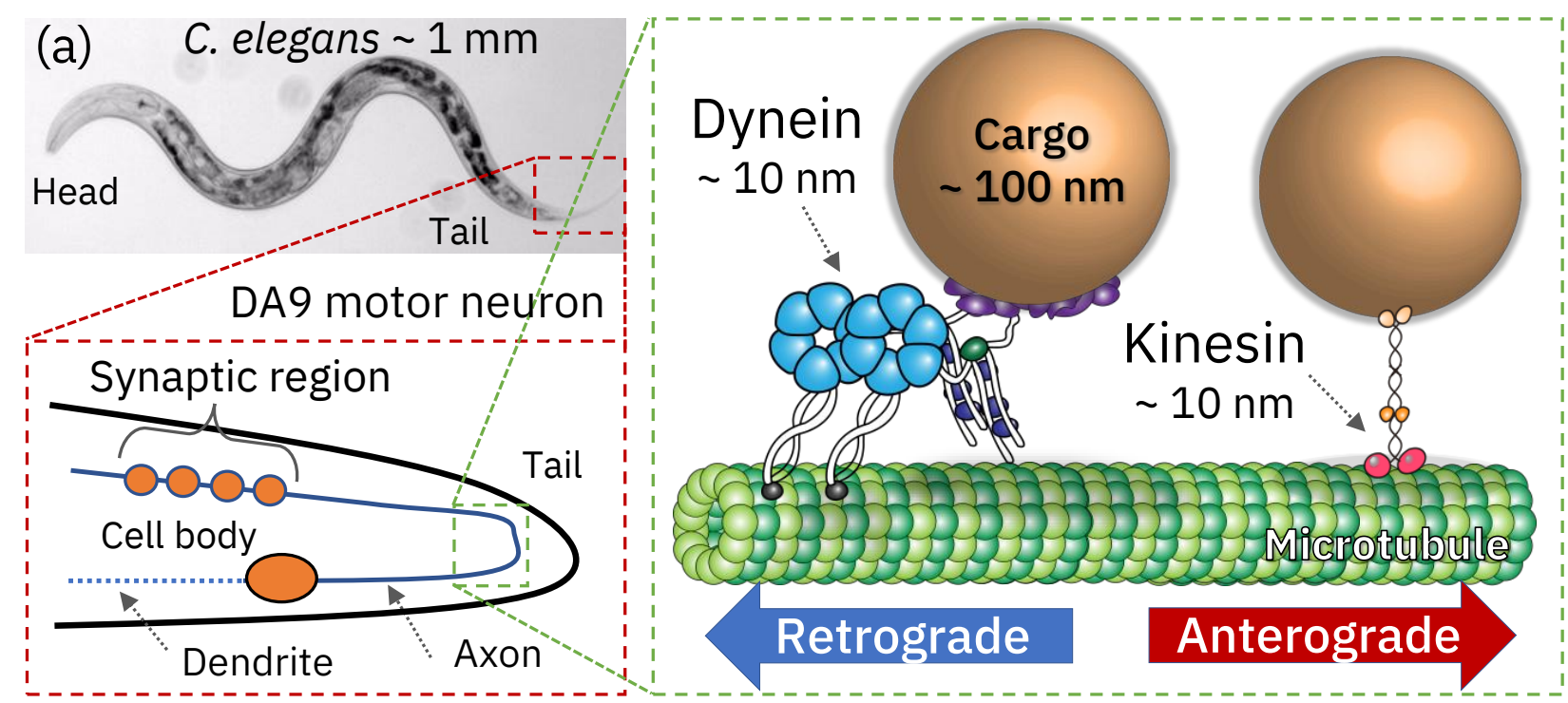

(b) Displacement along axon $(\mu \mathrm{m}) \Rightarrow$

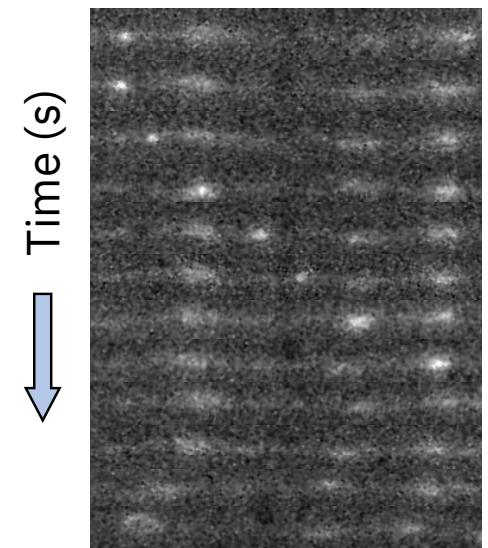

Recorded Images
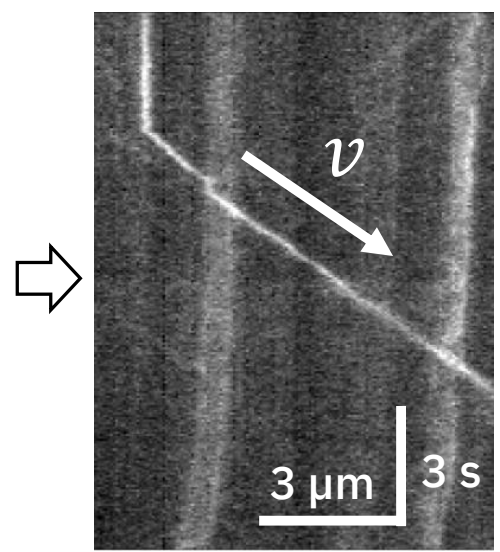

Kymograph (c) $1 2 0 0 \longdiv { n } = 1 3 6 1$

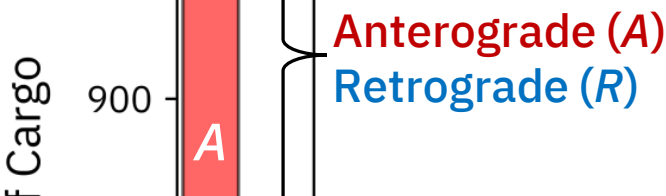
$\frac{1}{0}$
$\frac{1}{\Phi}$
$\frac{1}{E}$
$\frac{1}{z}$ 600 $300-\left\{\begin{array}{l}\text { With directional } \\ \text { changes } \\ A \rightarrow R \\ R \rightarrow A\end{array}\right.$

FIG. 1. (a) Schematics of anterograde and retrograde synaptic cargo transport by kinesin and dynein, respectively, in the DA9 motor neuron of a C. elegans worm. (b) Kymograph analysis. Velocity $(v)$ was measured as the slope of the trajectory of a fluorescent labeled cargo. (c) Number of synaptic cargos moving anterogradely (A), retrogradely (R), and exhibiting direction change $(A \rightarrow R$ and $R \rightarrow A)$, respectively. 

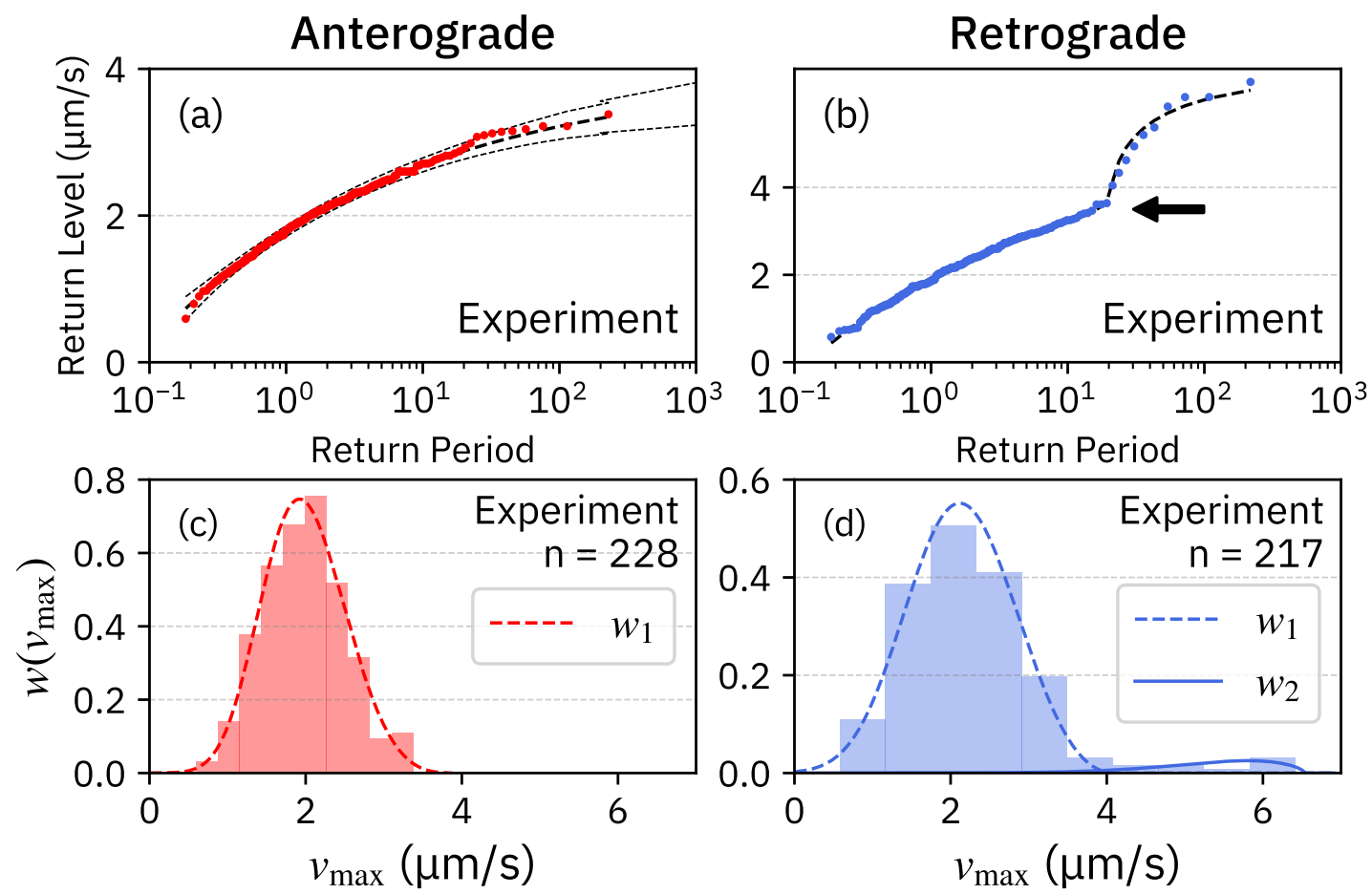

FIG. 2. Return level plots and probability distributions of transport velocity experimentally measured for anterograde $(a)(c)$ and retrograde transport $(b)(d)$, respectively. 

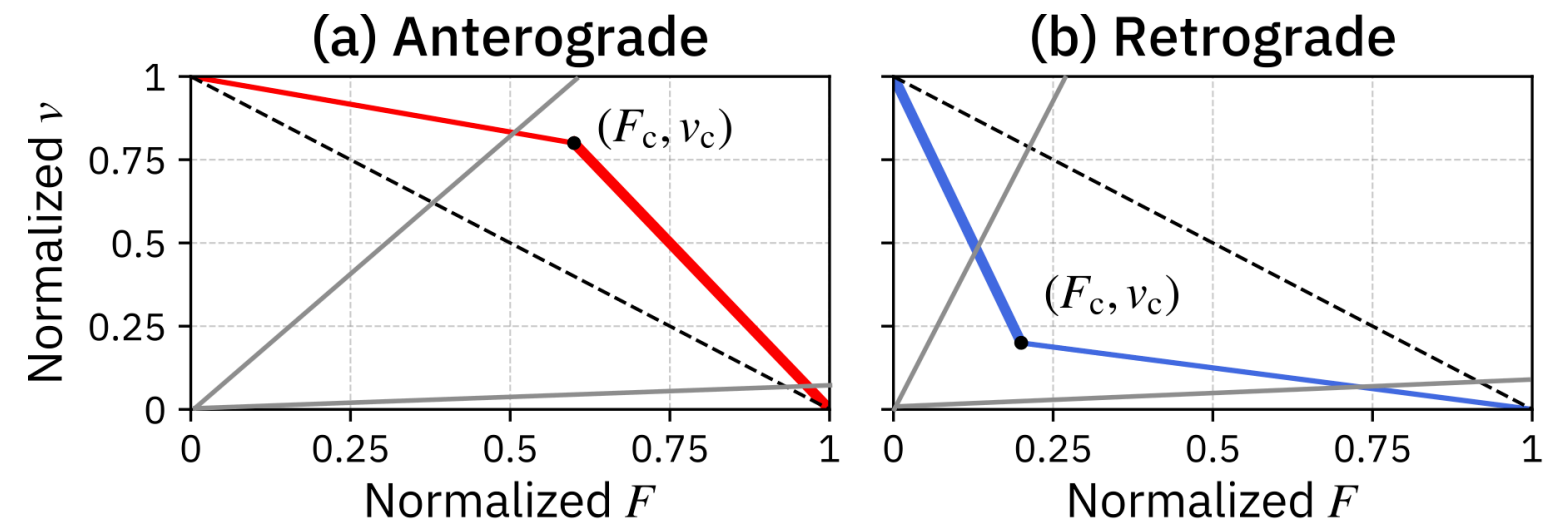

FIG. 3. Concave-down (a) and concave-up (b) force-velocity curves of a motor protein. Two regimes, load-sensitive (thick line) and load-insensitive (thin line) are represented. Gray lines of each graph represent $95 \%$ confidence interval of $\alpha$. Normalized $F: F / F_{\mathrm{S}}$ and $v: v / v(F=0)$, where $F_{\mathrm{S}}$ is the stall force of a motor protein. 

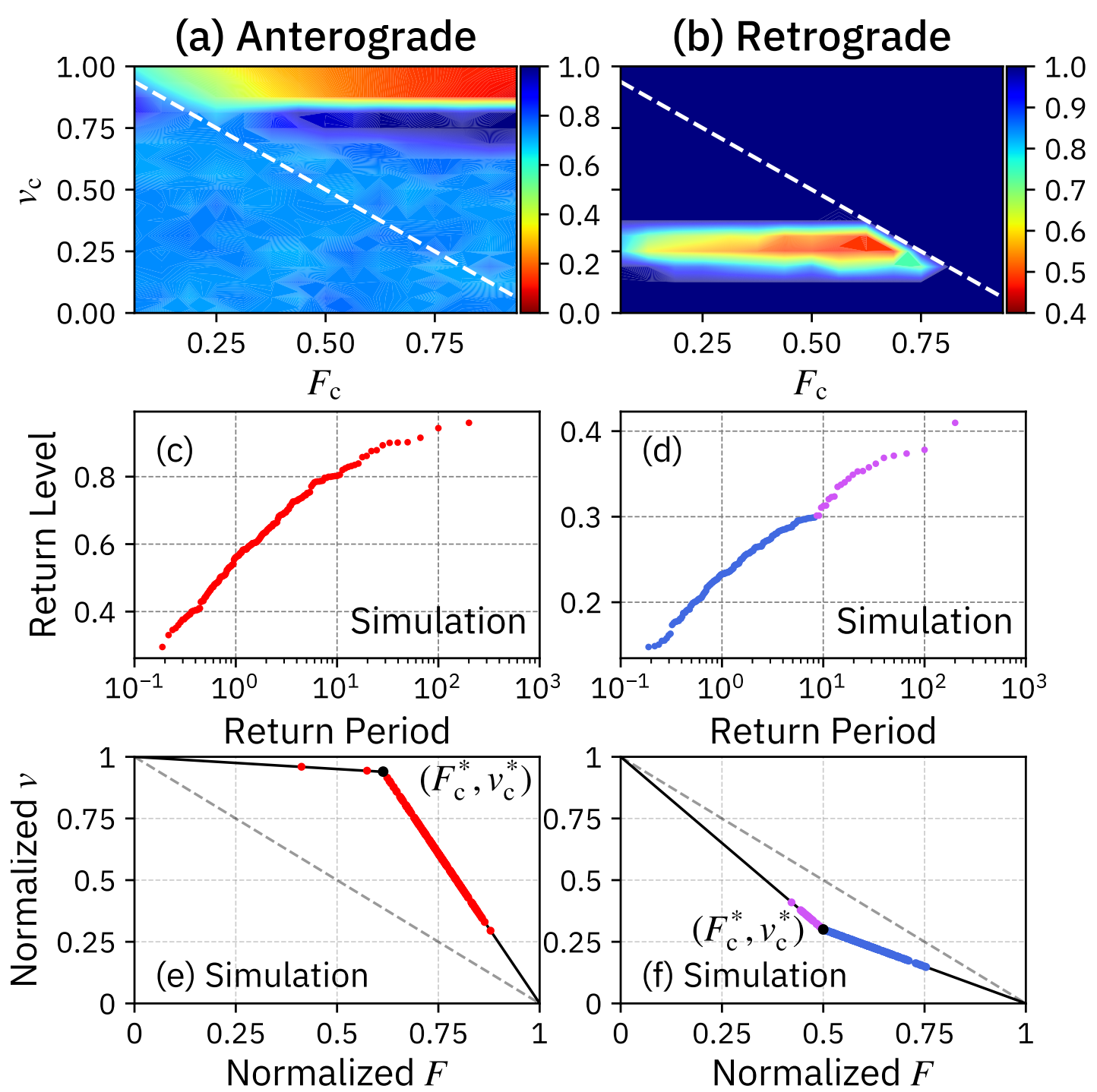

FIG. 4. Contour of $E\left(F_{c}, v_{c}\right)$, simulated return level plot, and force-velocity relationship obtained by using the most probable value of $\left(F_{\mathrm{c}}^{*}, v_{\mathrm{c}}^{*}\right)$ for anterograde $(\mathrm{a})(\mathrm{c})(\mathrm{e})$ and retrograde $(\mathrm{b})(\mathrm{d})(\mathrm{f})$ transport. Note that $y$ axis of Figs. 4(c) and 4(d) were normalized as $v / V_{\max }$. 


\section{REFERENCES}

[1] E. Gilleland and R. W. Katz, Journal of Software 72, 1 (2016).

[2] S. Coles, An Introduction to Statistical Modeling of Extreme Values, Springer, London (2001).

[3] L. de Haan and A. Ferreira, Extreme Value Theory, Springer (2006).

[4] M. Kratz, Matrix annals, 591 (2017).

[5] J. H. Einmahl and J. R. Magnus, J. Am. Stat. Assoc. 103, 1382 (2008).

[6] H. Rootzen and D. Zolud, Extremes 20, 713 (2017).

[7] H. Huxley and J. Hanson, Nature 173, 973 (1954).

[8] A. F. Huxley and R. Niedergerke, Nature 173, 971 (1954).

[9] N. Hirokawa, Y. Noda, Y. Tanaka, and S. Niwa, Nat. Rev. Mol. Cell Biol. 10, 682 (2009).

[10] R. D. Vale, Cell 112, 467 (2003).

[11] D. Okuno, R. Iino, and H. Noji, J. Biochem. 149, 655 (2011).

[12] M. J. Schnitzer, K. Visscher, and S. M. Block, Nat. Cell Biol. 2, 718 (2000).

[13] A. Gennerich, A. P. Carter, S. L. Reck-Peterson, and R. D. Vale, Cell 131, 952 (2007).

[14] S. Toba, T. M. Watanabe, L. Yamaguchi-Okimoto, Y. Y. Toyoshima, and H. Higuchi, Proc. Natl. Acad. Sci. USA 103, 5741 (2006).

[15] M. M. Elshenawy, J. T. Canty, L. Oster, L. S. Ferro, Z. Zhou, S. C. Blanchard, and A. Yildiz, Nat. Chem. Biol. 15, 1093 (2019).

[16] E. Hirakawa, H. Higuchi, and Y. Y. Toyoshima, Proc. Natl. Acad. Sci. USA 97, 2533 (2000).

[17] R. Mallik, B. C. Carter, S. A. Lex, S. J. King, and S. P. Gross, Nature 427, 649 (2004).

[18] D. H. Hall and E. M. Hedgecock, Cell 65, 837 (1991).

[19] A. J. Otsuka, A. Jeyaprakash, J. Garcia-Anoveros, L. Z. Tang, G. Fisk, T. Hartshorne, R. Franco, and T. Born, Neuron 6, 113 (1991).

[20] C. W. Chen, Y. F. Peng, Y. C. Yen, P. Bhan, M. Muthaiyan Shanmugam, D. R. Klopfenstein, and O. I. Wagner, J. Neurosci. Res. 97, 185 (2019).

[21] K. Hayashi, S. Hasegawa, T. Sagawa, S. Tasaki, and S. Niwa, Phys. Chem. Chem. Phys. 20, 3403 (2018).

[22] See Supplemetary Material for detailed materials and methods, supplementary figures.

[23] W. S. Rasband, National Institute of Health, Bethesda, Maryland, USA, http://imagej.nih.gov/ji/ (1997).

[24] S. P. Gross, Phys. Biol. 1, R1 (2004).

[25] R Core Team, Vienna, Austria, https://www.R-project.org/ (2018).

[26] R. A. Longoria and G. T. Shubeita, PLoS One 8, e67710 (2013).

[27] A. K. Rai, A. Rai, A. J. Ramaiya, R. Jha, and R. Mallik, Cell 152, 172 (2013).

[28] K. Hayashi, M. G. Miyamoto, and S. Niwa, Biophys. J. 120, 1605 (2021). 
[29] K. Hayashi, Y. Tsuchizawa, M. Iwaki, and Y. Okada, Mol. Biol. Cell, mbcE18010022 (2018).

[30] S. Hasegawa, T. Sagawa, K. Ikeda, Y. Okada, and K. Hayashi, Sci. Rep. 9, 5099 (2019).

[31] K. Sasaki, M. Kaya, and H. Higuchi, Biophys. J. 115, 1981 (2018).

[32] K. Hayashi, S. Matsumoto, T. Naoi, and Y. Idobata, preprint, bioRxiv:2021.09.12.459977v4.

[33] B. G. Budaitis, S. Jariwala, L. Rao, Y. Yue, D. Sept, K. J. Verhey, and A. Gennerich, J. Cell Biol. 220 (2021).

[34] Y. Anazawa, T. Kita, K. Hayashi, and S. Niwa, bioRxiv:2021.07.22.453457v2.

[35] D. R. Gabrych, V. Z. Lau, S. Niwa, and M. A. Silverman, Front. Cell Neurosci. 13, 419 (2019). 


\section{Supplemental Material}

\section{Materials and Methods}

Sample preparation. In our study, we used C. elegans stains wyIs251[Pmig-13::gfp::rab-3; Podr-1::gfp]; wyIs 251 has been previously described $(1,2)$.

Culture. C. elegans was maintained on OP50 feeder bacteria on nematode agar plates (NGM) agar plates, as per the standard protocol $(1,2)$. The strains were maintained at $20^{\circ} \mathrm{C}$.

Fluorescence microscopy observations. A cover glass $(32 \mathrm{~mm} \times 24 \mathrm{~mm}$, Matsunami Glass Ind., Ltd., Tokyo, Japan) was coated with 10\% agar (Wako, Osaka, Japan). A volume of $20 \mu \mathrm{L}$ of $25 \mathrm{mM}$ levamisole mixed with $5 \mu \mathrm{L} 200$-nm-sized polystyrene beads (Polysciences Inc., Warrington, PA, USA) was dropped onto the cover glass. The polystyrene beads increased the friction and inhibited the movement of worms; levamisole paralyzed the worms. Ten to twenty worms were transferred from the culture dish to the medium on the cover glass. A second cover glass was placed over the first cover glass forming a chamber, thereby confining the worms. The worms in the chamber were observed under a fluorescence microscope (IX83, Olympus, Tokyo, Japan) at room temperature. Images of a GFP (green fluorescence protein)-labelled synaptic cargos in the DA9 motor neuron were obtained using a $150 \times$ objective lens (UApoN 150x/1.45, Olympus) and an sCMOS camera (OLCA-Flash4.0 V2, Hamamatsu Photonics, Hamamatsu, Japan) at 10 frames per second.

\section{Cargo size distribution}

The fluorescence images of various synaptic cargos are shown in Fig. S1. The difference in fluorescence intensity represents the difference in the sizes of cargos. This is because fluorescence proteins are uniformly distributed on the surface of a cargo and FI $\propto 4 \pi r^{2}$ ( $r$ : radius of a cargo). Anterograde and retrograde transports were not distinguished in the analysis of FI.

The distribution of $x=1 / \sqrt{\mathrm{FI}}$ is well fitted by a Gamma distribution

$$
f(x ; k, \lambda)=\frac{\lambda^{k}}{\Gamma(k, \lambda)} x^{k-1} e^{-\lambda x}
$$

where $\Gamma$ is a Gamma function, $k=6.54 \pm 0.78$ and $\lambda=201 \pm 25$ (the errors are fitting errors).

\section{Histogram of velocity}

Histograms of measured velocities $\left\{v^{i}\right\} \quad(i=1, \cdots, n$ where $n=2091$ for anterograde transport and $n=1113$ for retrograde transport) for anterograde and retrograde transport are shown in Fig. S2. The mean velocities were $1.57 \pm 0.45$ (SE) $\mu \mathrm{m} / \mathrm{s}$ and $1.78 \pm 0.72(\mathrm{SE}) \mu \mathrm{m} / \mathrm{s}$ for anterograde and retrograde transport, respectively.

\section{Error of $V_{\text {max }}$}

The mean $\left(\overline{V_{\max }}\right)$ and error $\left(\delta V_{\max }\right)$ of $V_{\max }$ were estimated from the fitting parameters $\mu(=\bar{\mu} \pm \delta \mu)$, 
$\sigma(=\bar{\sigma} \pm \delta \sigma)$ and $\xi(=\bar{\xi} \pm \delta \xi)$ of the Weibull distributions defined by Eq. (1) in the main text as follows:

$$
V_{\max }=\left(\bar{\mu}-\frac{\bar{\sigma}}{\bar{\xi}}\right) \pm \sqrt{(\delta \mu)^{2}+\left(\frac{\delta \sigma}{\bar{\xi}}\right)^{2}+\left(\frac{\bar{\sigma} \delta \xi}{\bar{\xi}^{2}}\right)^{2}} .
$$

The values of $\mu(=\bar{\mu} \pm \delta \mu), \sigma(=\bar{\sigma} \pm \delta \sigma)$ and $\xi(=\bar{\xi} \pm \delta \xi)$ for anterograde and retrograde transport are summarized in Table $\mathrm{S} 1$.

\section{Bootstrapping method}

A part ( $r$ : ratio) of the data $\left\{v_{\max }^{i}\right\}(i=1, \cdots, N)$ was randomly selected, and then the fitting parameters of $\mu$, $\sigma$ and $\xi$ of the Weibull distributions were calculated for the part. Here, duplication of the same data was allowed if it was selected. This procedure was repeated 100 times, and then the errors of $\mu, \sigma$ and $\xi$ were calculated. In Fig. S3, each parameter is plotted as a function of $r$ in the cases of anterograde and retrograde transport. The values were stable for a wide range of $r(0.4 \leq r \leq 1)$.

\section{Block size}

We investigated the dependence of the fitting parameters $(\mu, \sigma$ and $\xi)$ on the number $(s)$ of worms (block size), from which $v_{\max }^{i}$ was chosen, as shown in Fig. S4. All values converge as $s$ increases.

\section{Simulation}

\section{Multiple motors}

It has been suggested that a single cargo is transported by multiple motors. We used the force-velocity relationships depicted in Fig. S5 to investigate the effect of multiple motors on the error function (Eq. (3) in the main text); the blue line, orange line and green line in Fig. S5 represent the force-velocity relationship of the transport by one motor, by two motors, or by three motors, respectively. In the simulation, the case of one motor or that of two motors, and that of three motors was chosen according to the appearance ratio 1:2:1, which was determined in our previous study (3). For example, when three motors (represented by the green line in Fig. $\mathrm{S} 5$ left) are chosen, $v_{\text {sim }}^{i}$ is determined as the intersection between the green line and the straight line represents the Stokes' law.

\section{Tug-of-war}

The effect of the tug-of-war between opposing motors was modeled as follows: a moving cargo is sometimes (probability: $p$ ) subject to the constant load $F_{\text {TOW }}$ added to the viscous load $(\Gamma v)$ calculated, where $\Gamma$ was the friction coefficient of the cargo, from the force velocity model (Fig. 3 in the main text). The load from the opposing motor $F_{\text {TOW }}$ was set to $20 \%$ of the stall force $F_{\mathrm{S}}$ of the motor. The calculation results of the error function (Eq. (3) of the main text) are shown for the anterograde (Fig. S6) and retrograde (Fig. S7) transport. 


\section{Chemo-mechanical model}

The chemo-mechanical coupling models of kinesin and dynein were theoretically derived based on the ATP hydrolysis mechanisms of motor proteins. These models provide the force-velocity relationships. The forcevelocity relationship for the one-state model of dynein (4) is represented as

$$
v(F)=v_{\min }\left(1-e^{-\left(F-F_{\mathrm{S}}\right) d / k_{\mathrm{B}} T}\right),
$$

where $k_{\mathrm{B}}, T$ and $d$ are the Boltzmann constant, the temperature of the environment, and the characteristic distance, respectively (see Ref. (4) for definitions of parameters). The simulated return level plot obtained by using the force-velocity relationship (Eq. (S2)) is shown in Fig. S8. As a more realistic model, a three state model was developed for both kinesin and dynein (5). The force-velocity relationship for the three-state model is represented as

$$
\begin{gathered}
v(F)=\left(k_{01}-k_{02}\right) l, \\
\frac{1}{k_{01}}=\frac{1}{\kappa_{1}}+\frac{1}{\lambda_{1}} e^{d_{1} F / k_{\mathrm{B}} T}, \\
\frac{1}{k_{02}}=\frac{1}{\lambda_{2}} e^{-d_{2} F / k_{\mathrm{B}} T},
\end{gathered}
$$

(see Ref. (5) for the definitions of parameters). The simulated return level plots obtained by using the force-velocity relationship (Eq. (S3)) were generated for the anterograde (Fig. S9) and retrograde (S10) transports. Finally, the force-velocity relationship for the energy landscape model of kinesin (6) is represented as

$$
\begin{gathered}
v(F)=\frac{d}{\frac{1}{k_{\mathrm{cat}}}+\frac{1}{k_{\mathrm{b}}}[\mathrm{ATP}]}, \\
k_{\mathrm{cat}}=\frac{k_{\mathrm{cat}}^{0}}{1-q_{\mathrm{cat}}\left(1-e^{F \delta / k_{\mathrm{B}} T}\right)}, \\
k_{\mathrm{b}}=\frac{k_{\mathrm{b}}^{0}}{1-q_{\mathrm{b}}\left(1-e^{F \delta / k_{\mathrm{B}} T}\right)},
\end{gathered}
$$

where [ATP] is the concentration of ATP (see Ref. (6) for the definitions of parameters). The simulated return level plot obtained by using the force-velocity relationship (Eq. (S4)) is plotted in Fig. S11. The tendency of the return level plots using the above models was similar to that of the return level plots (Fig. 4(c) and 4(d) in the main text) obtained by using the simple models (Fig. 3 in the main text). The parameters used in Fig. S8-S11 are summarized in Table S2.

\section{Synaptic cargo transport in mouse hippocampal neurons}

In the investigation of mammalian dynein, EVA was also applied to data of synaptic cargo transport in mouse hippocampal neurons, originally obtained in Ref. (7). The values of $\mu(=\bar{\mu} \pm \delta \mu), \sigma(=\bar{\sigma} \pm \delta \sigma)$ and $\xi(=$ $\bar{\xi} \pm \delta \xi$ ) for anterograde and retrograde transport are summarized in Table S1. 


\section{Reference}

1. Niwa, S., D. M. Lipton, M. Morikawa, C. Zhao, N. Hirokawa, H. Lu, and K. Shen. 2016. Autoinhibition of a Neuronal Kinesin UNC-104/KIF1A Regulates the Size and Density of Synapses. Cell Rep 16(8):2129-2141.

2. Wu, Y. E., L. Huo, C. I. Maeder, W. Feng, and K. Shen. 2013. The balance between capture and dissociation of presynaptic proteins controls the spatial distribution of synapses. Neuron 78(6):9941011.

3. Hayashi, K., S. Hasegawa, T. Sagawa, S. Tasaki, and S. Niwa. 2018. Non-invasive force measurement reveals the number of active kinesins on a synaptic vesicle precursor in axonal transport regulated by ARL-8. Phys Chem Chem Phys 20(5):3403-3410.

4. Elshenawy, M. M., J. T. Canty, L. Oster, L. S. Ferro, Z. Zhou, S. C. Blanchard, and A. Yildiz. 2019. Cargo adaptors regulate stepping and force generation of mammalian dynein-dynactin. Nat Chem Biol 15(11):1093-1101.

5. Sasaki, K., M. Kaya, and H. Higuchi. 2018. A Unified Walking Model for Dimeric Motor Proteins. Biophys J 115(10):1981-1992.

6. Schnitzer, M. J., K. Visscher, and S. M. Block. 2000. Force production by single kinesin motors. Nat Cell Biol 2(10):718-723.

7. Hayashi, K., M. G. Miyamoto, and S. Niwa. 2021. Effects of dynein inhibitor on the number of motor proteins transporting synaptic cargos. Biophys J 120(9):1605-1614. 

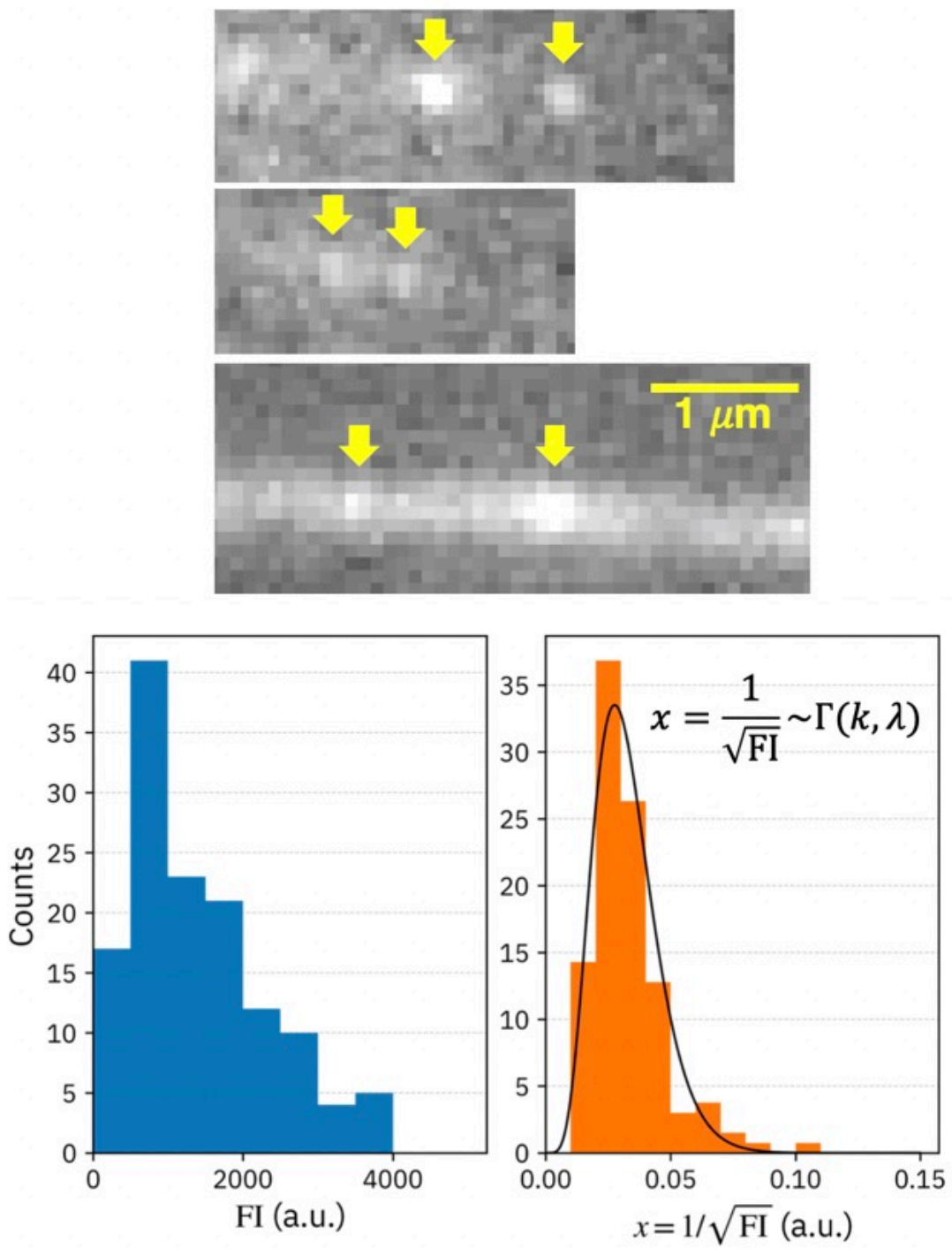

Fig. S1 (Top) Examples of fluorescence micrographs of synaptic cargos transported by motor proteins in the axons of DA9 neurons of C. elegans worms. (Bottom) Fluorescence intensity (FI) of synaptic cargos $(n=133)$ (left panel). The distribution of $x=1 / \sqrt{\mathrm{FI}}$ was fitted by a Gamma distribution (right panel). 

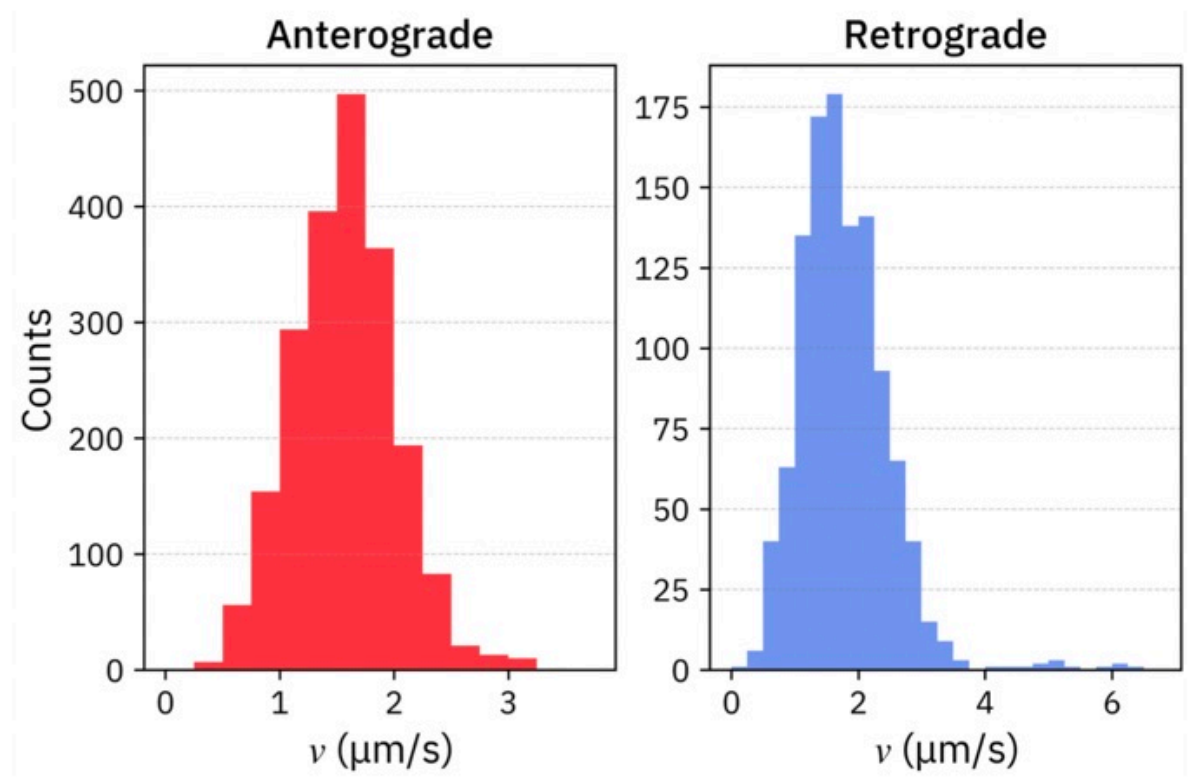

Fig. S2 Histogram of velocity $\left\{v^{i}\right\}$ of synaptic cargos for anterograde (red) and retrograde (blue) transport. 


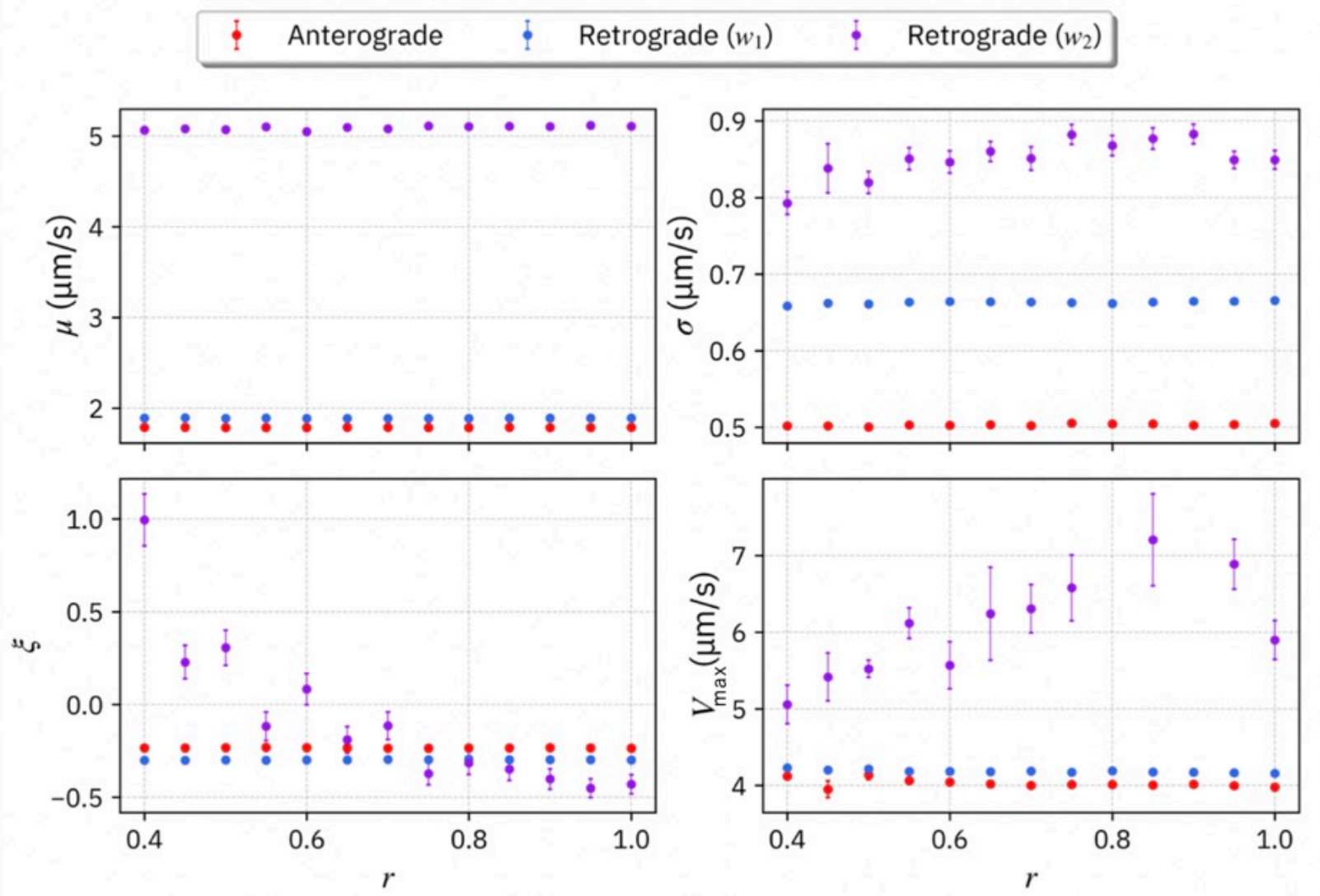

Fig. S3 Fitting parameters of $\mu, \sigma$, and $\xi$ plotted as a function of $r$. The bootstrapping analysis was repeated 100 times for each $r$. The error-bars represent the statistical errors (SE). 


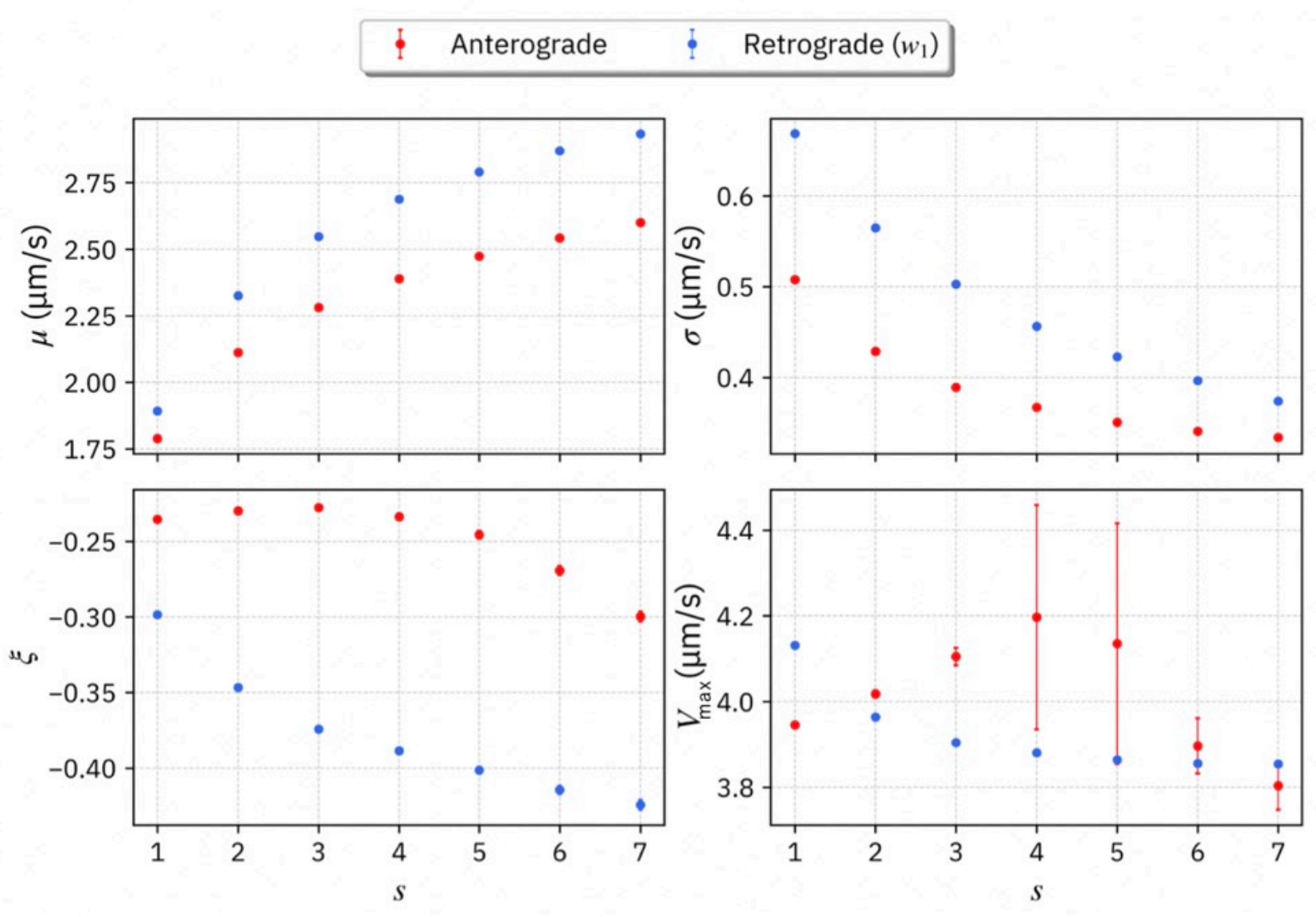

Fig. S4 Fitting parameters of $\mu, \sigma$, and $\xi$ plotted as a function of the number $(s)$ of worms (block size). The error-bars represent the statistical errors (SE) for the 100 repeats of bootstrapping trials $(r=1)$. Note that it was difficult to investigate $s$ dependence in the retrograde case $\left(w_{2}\right)$ because of the small size of the data. 


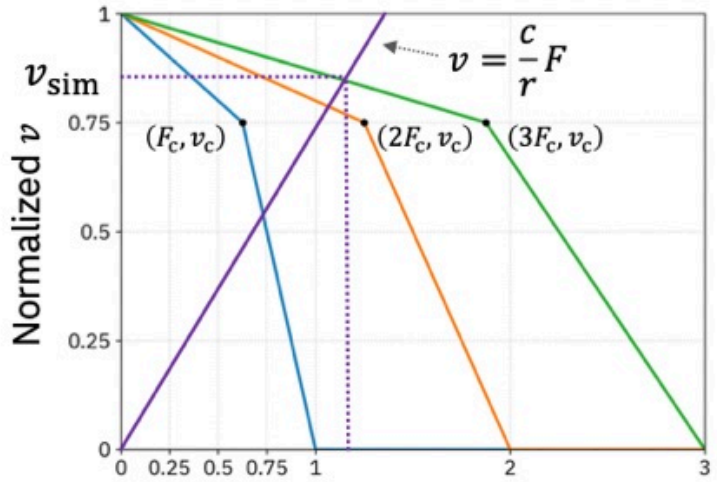

Normalized $F$
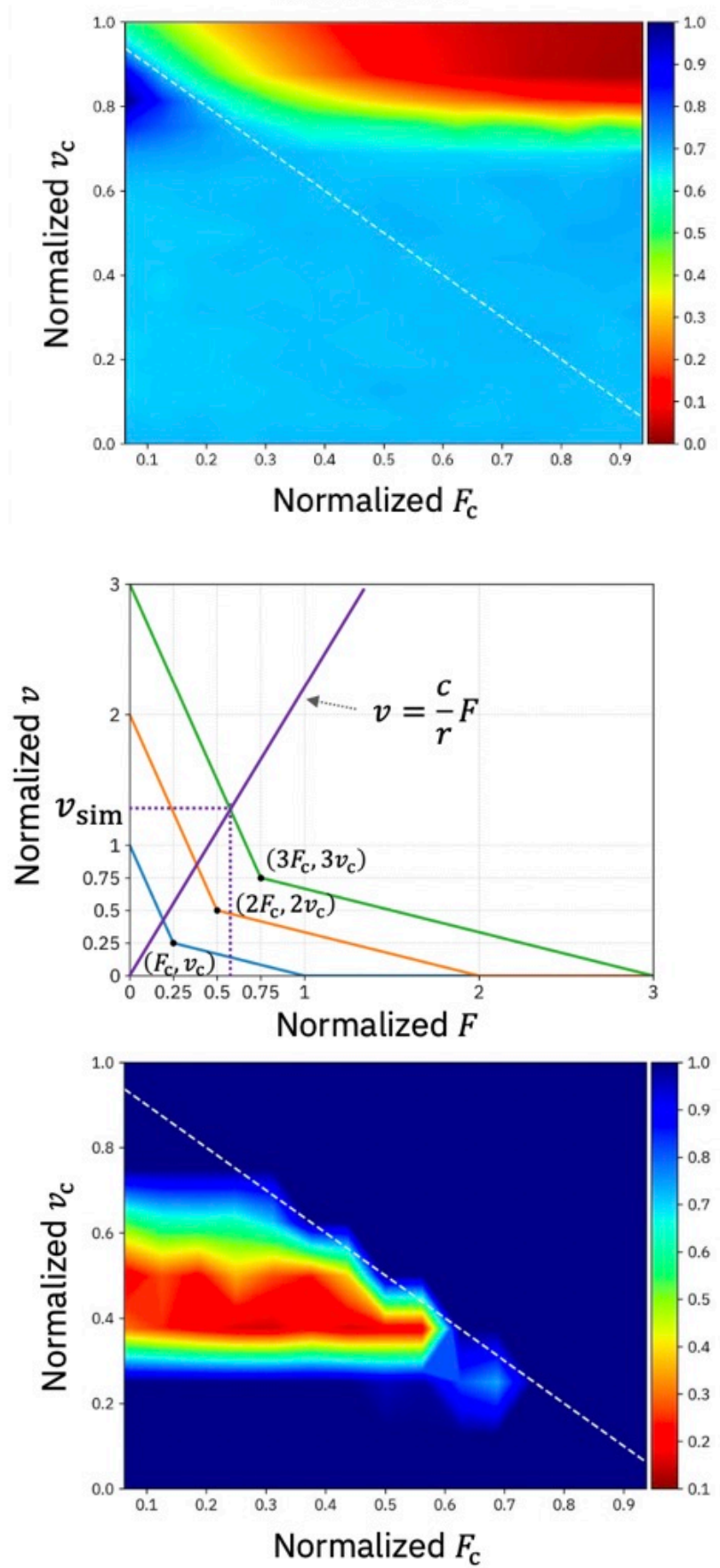

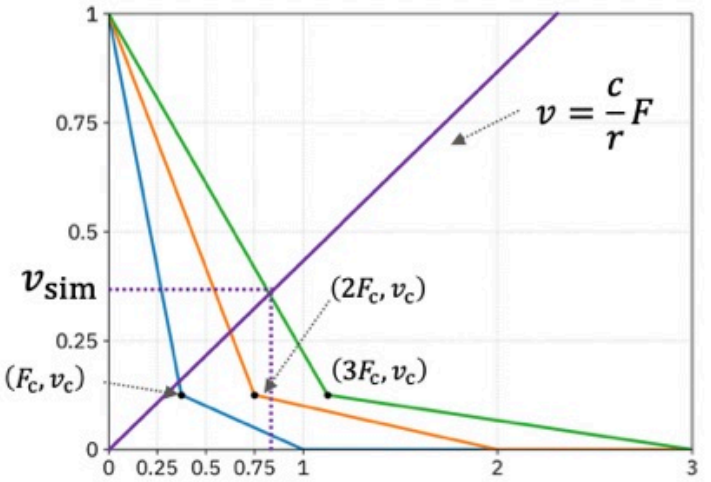

Normalized $F$
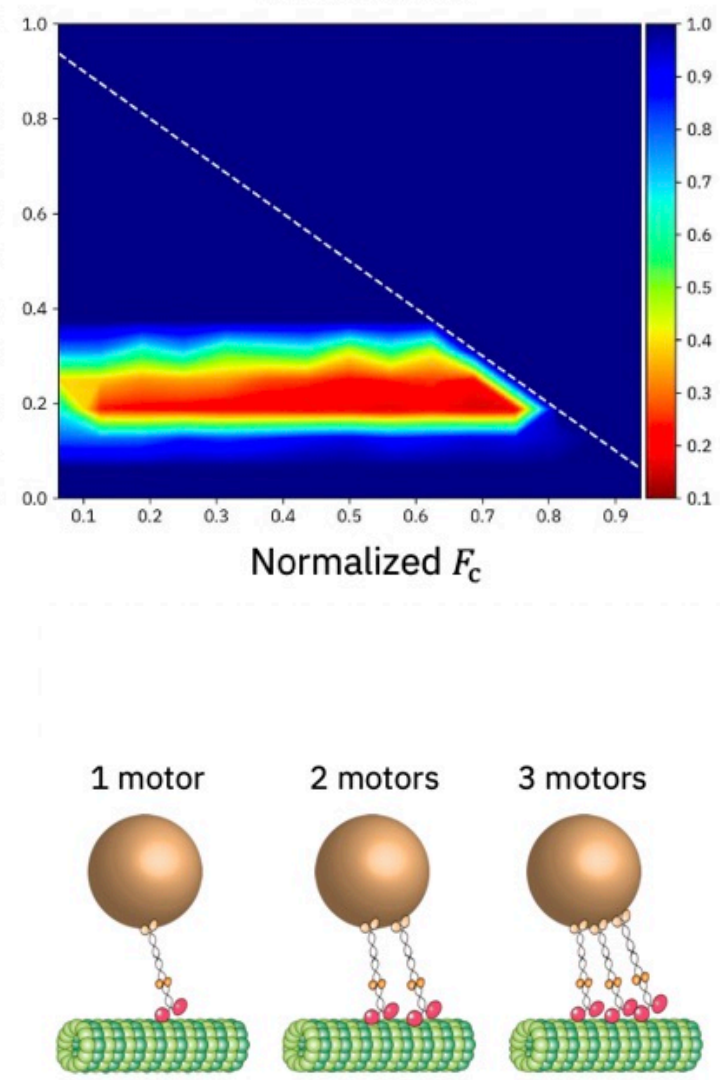

Fig. S5 Force-velocity model and the error function (Eq. (3) in the main text) in the case of cargo transport by multiple motors for anterograde (top left panels) and retrograde transport (top right and bottom left panels). The blue line, orange line, and green line represent the cases of transport by one motor, that by two motors, and that by three motors, respectively. 
bioRxiv preprint doi: https://doi.org/10.1101/2021.12.29.474400; this version posted December 30, 2021. The copyright holder for this preprint (which was not certified by peer review) is the author/funder, who has granted bioRxiv a license to display the preprint in perpetuity. It is made available under aCC-BY-NC-ND 4.0 International license.

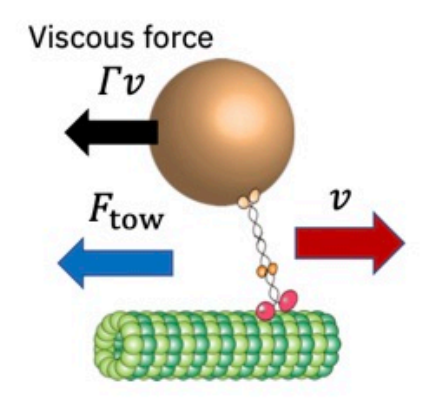

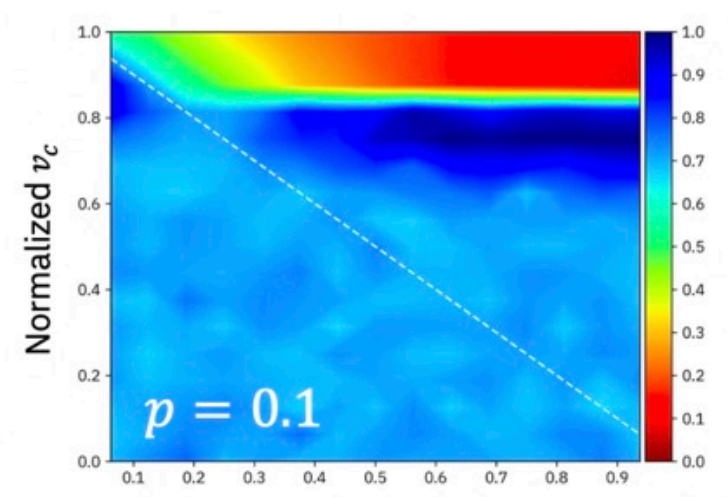

Normalized $F_{c}$

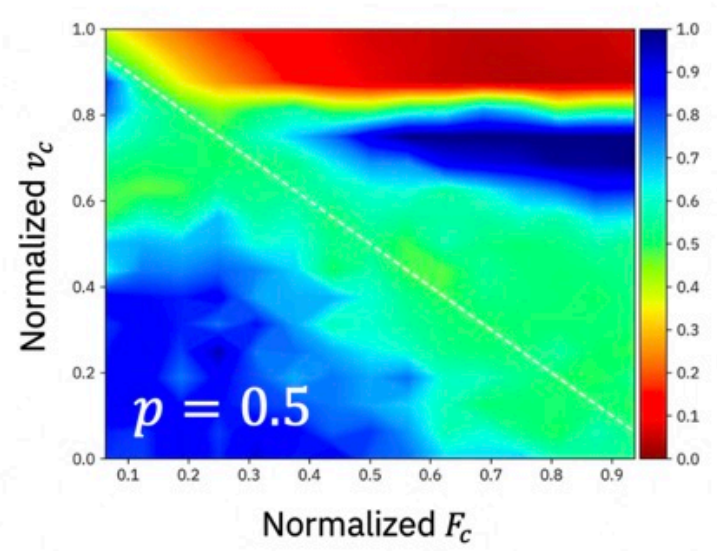

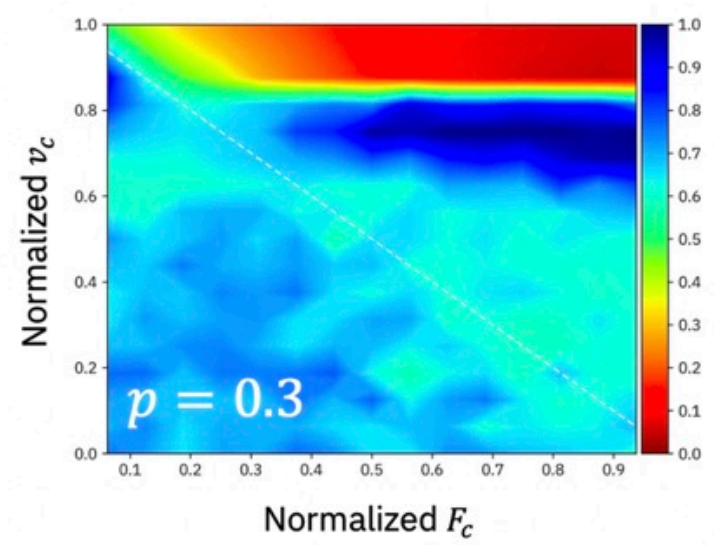

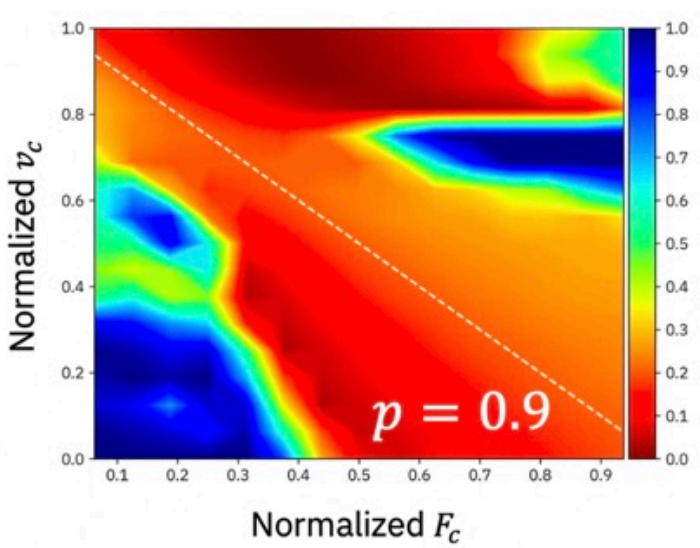

Fig. S6 Contour of the error function $E\left(F_{\mathrm{c}}, v_{\mathrm{c}}\right)$ for anterograde transport in the cases of $p=$ $0.1,0.3,0.5,0.9$. 
bioRxiv preprint doi: https://doi.org/10.1101/2021.12.29.474400; this version posted December 30, 2021. The copyright holder for this preprint (which was not certified by peer review) is the author/funder, who has granted bioRxiv a license to display the preprint in perpetuity. It is made available under aCC-BY-NC-ND 4.0 International license.
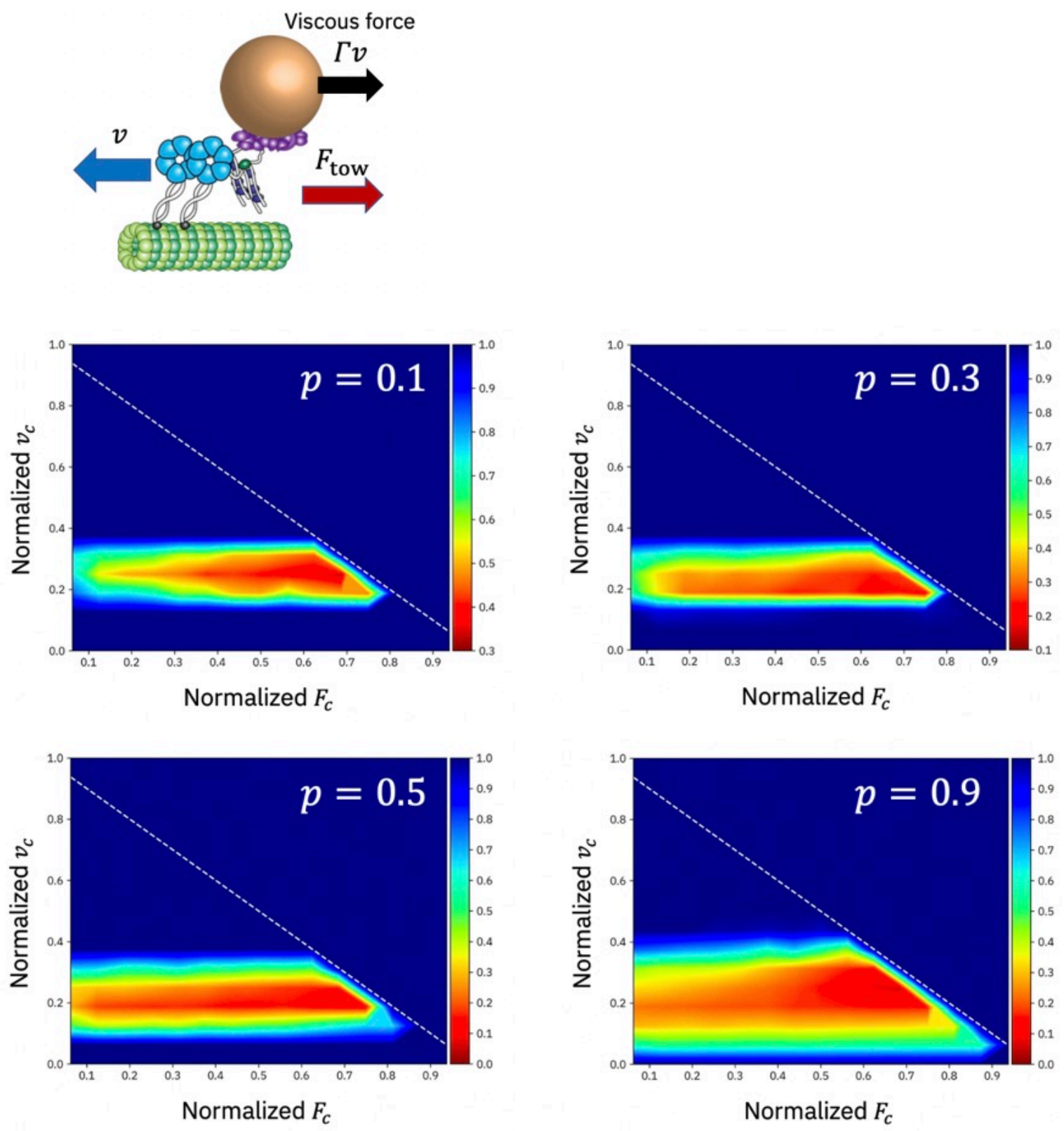

Fig. S7 Contour of the error function $E\left(F_{\mathrm{c}}, v_{\mathrm{c}}\right)$ for retrograde transport in the cases of $p=0.1,0.3,0.5,0.9$. 
bioRxiv preprint doi: https://doi.org/10.1101/2021.12.29.474400; this version posted December 30, 2021. The copyright holder for this preprint (which was not certified by peer review) is the author/funder, who has granted bioRxiv a license to display the preprint in perpetuity. It is made available under aCC-BY-NC-ND 4.0 International license.
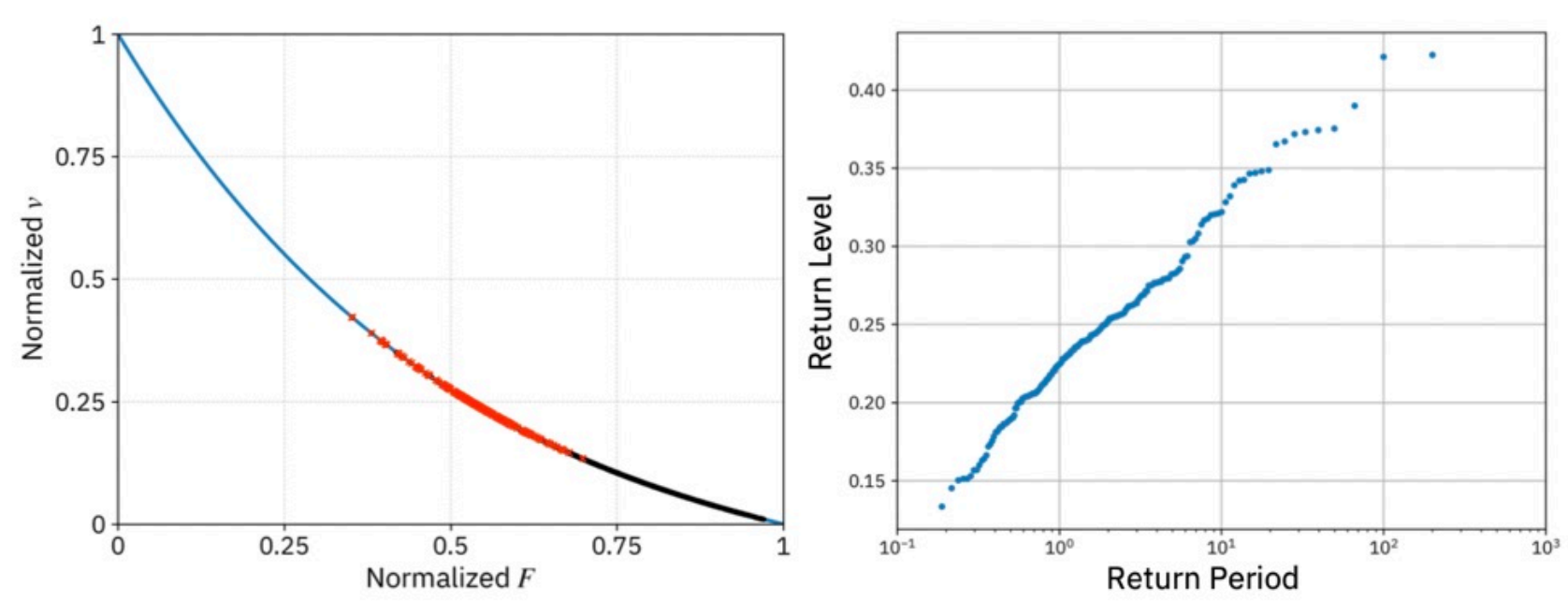

Fig. S8 Force-velocity relationship of the one-state model for dynein (4). The red symbols represent $\left\{v_{\text {sim,max }}^{i}\right\}$. The corresponding simulated return level plot (right panel). 

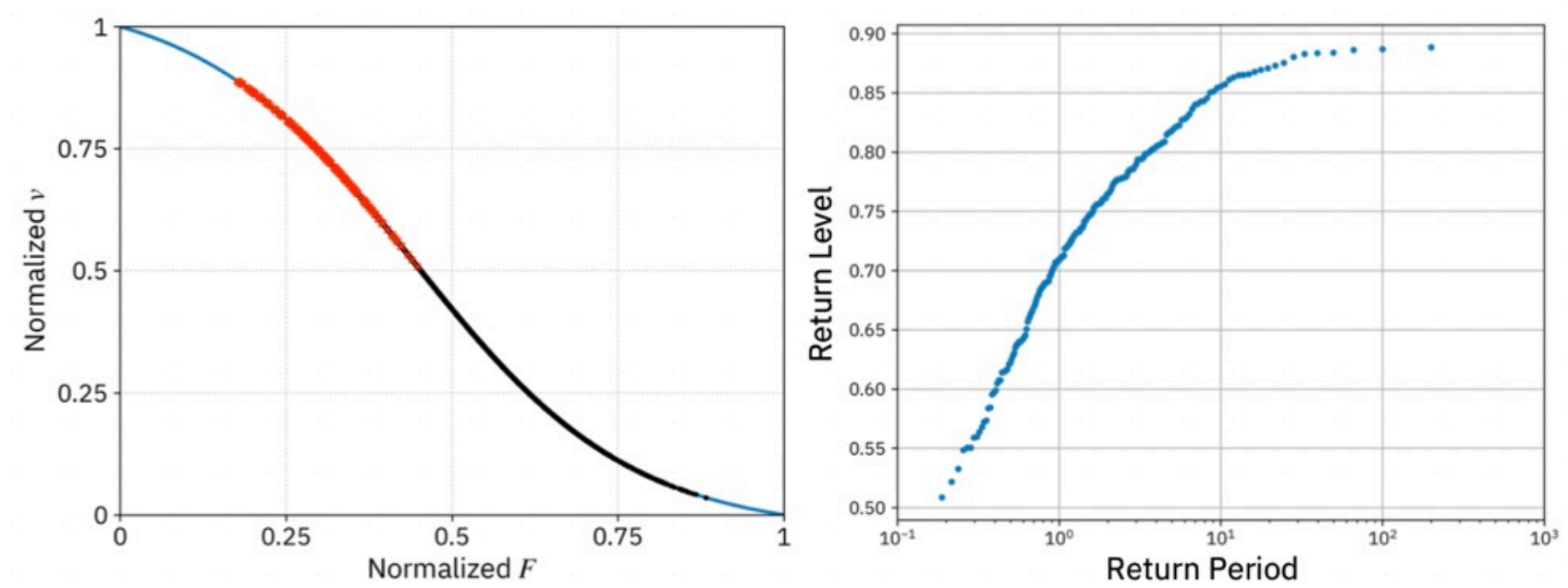

Fig. S9 Force-velocity relationship of the three-state model for kinesin (5). The red symbols represent $\left\{v_{\text {sim,max }}^{i}\right\}$. The corresponding simulated return level plot (right panel).
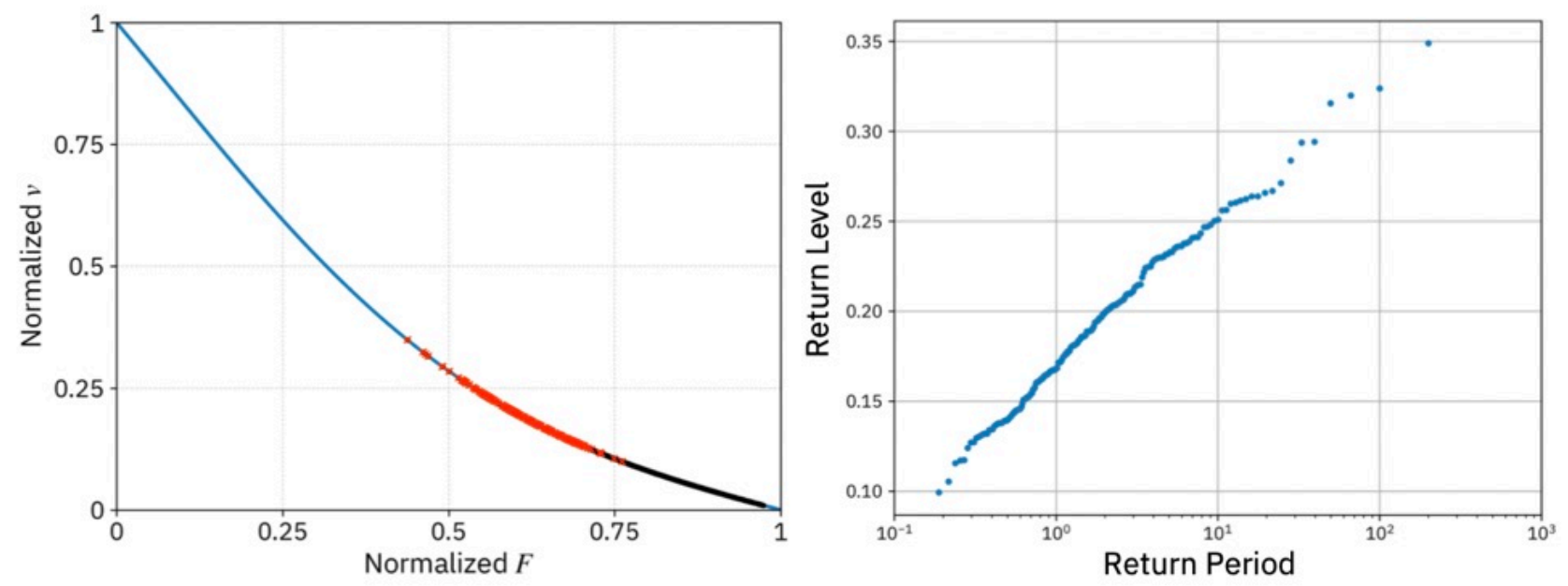

Fig. S10 Force-velocity relationship of the three-state model for dynein (5). The red symbols represent $\left\{v_{\text {sim,max }}^{i}\right\}$. The corresponding simulated return level plot (right panel). 
bioRxiv preprint doi: https://doi.org/10.1101/2021.12.29.474400; this version posted December 30, 2021. The copyright holder for this preprint (which was not certified by peer review) is the author/funder, who has granted bioRxiv a license to display the preprint in perpetuity. It is made available under aCC-BY-NC-ND 4.0 International license.
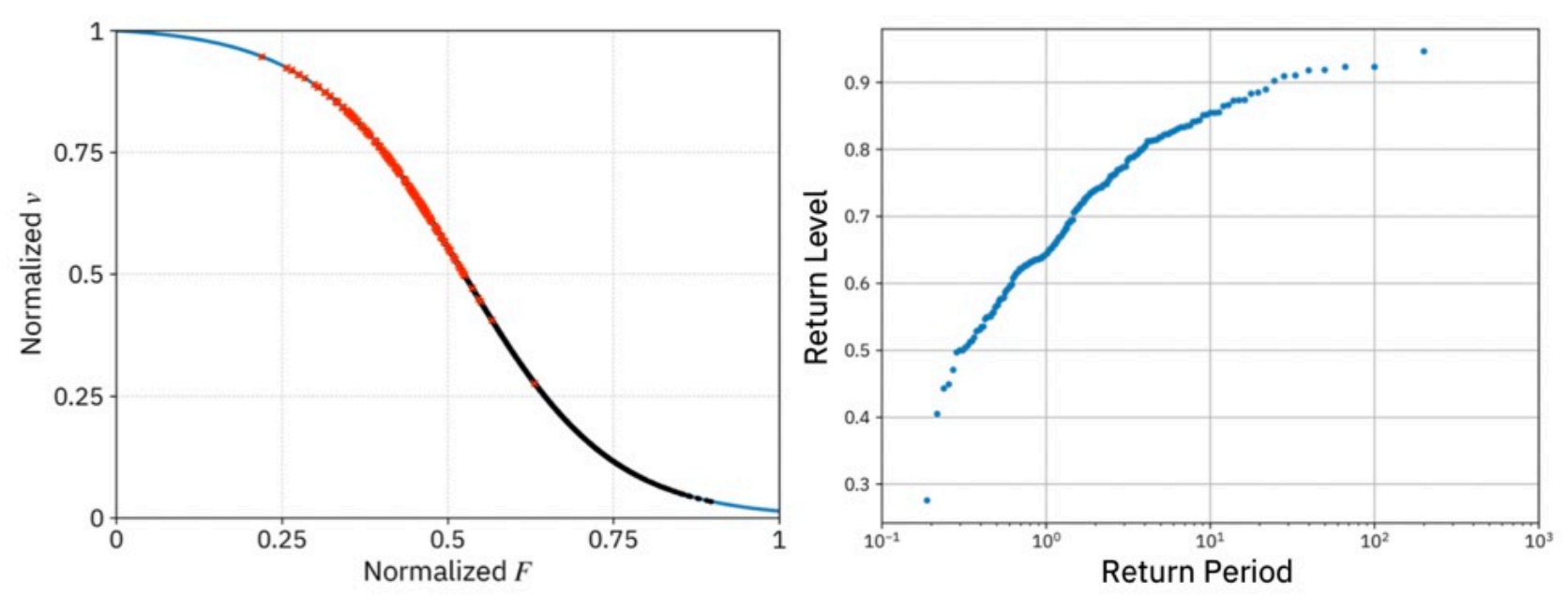

Fig. S11 Force-velocity relationship of the energy landscape model for dynein (6). The red symbols represent $\left\{v_{\text {sim,max }}^{i}\right\}$. The corresponding simulated return level plot (right panel). 

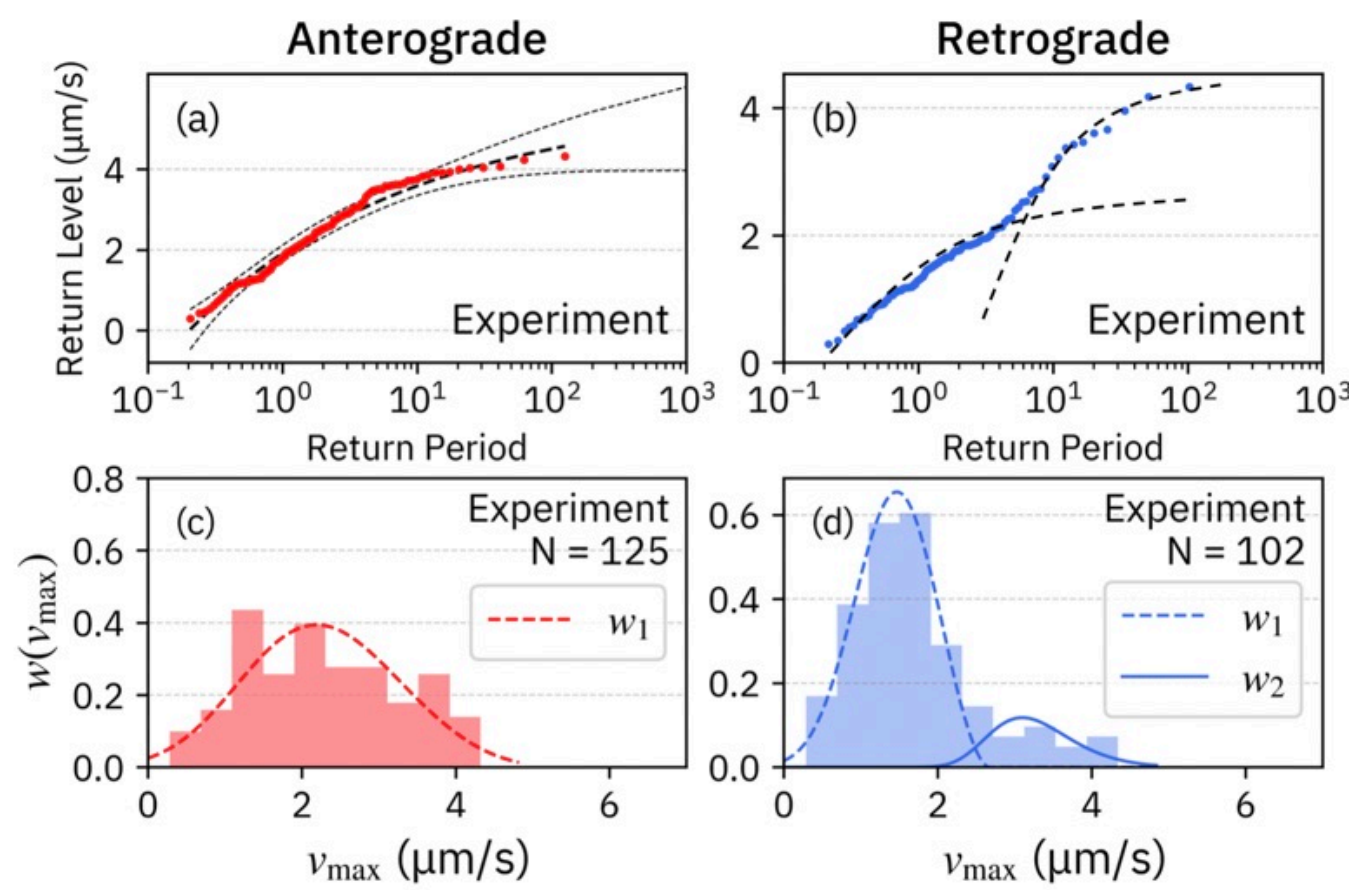

Fig. S12 Return level plot and probability distributions of transport velocity experimentally measured for anterograde (a)(c) and retrograde transport (b)(d) in the case of synaptic cargo transport in mouse hippocampal neurons, originally obtained in Ref. (7). 


\begin{tabular}{c|c|c|c}
\hline C.elegans & Anterograde & Retrograde $\left(w_{1}\right)$ & Retrograde $\left(w_{2}\right)$ \\
\hline$\mu(\mu \mathrm{m} / \mathrm{s})$ & $1.79 \pm 0.04$ & $1.89 \pm 0.05$ & $4.98 \pm 0.32$ \\
\hline$\sigma(\mu \mathrm{m} / \mathrm{s})$ & $0.508 \pm 0.026$ & $0.668 \pm 0.037$ & $0.780 \pm 0.262$ \\
\hline$\xi$ & $-0.235 \pm 0.042$ & $-0.299 \pm 0.049$ & $-0.400 \pm 0.416$ \\
\hline$V_{\max }(\mu \mathrm{m} / \mathrm{s})$ & $3.95 \pm 0.40$ & $4.13 \pm 0.39$ & $6.53 \pm 0.99$ \\
\hline Mammalian $($ Mouse $)$ & Anterograde & Retrograde $\left(w_{1}\right)$ & Retrograde $\left(w_{2}\right)$ \\
\hline$\mu(\mu \mathrm{m} / \mathrm{s})$ & $1.91 \pm 0.10$ & $1.25 \pm 0.06$ & $3.34 \pm 0.09$ \\
\hline$\sigma(\mu \mathrm{m} / \mathrm{s})$ & $0.970 \pm 0.078$ & $0.530 \pm 0.044$ & $0.233 \pm 0.068$ \\
\hline$\xi$ & $-0.261 \pm 0.093$ & $-0.191 \pm 0.075$ & $-0.172 \pm 0.301$ \\
\hline$V_{\max }(\mu \mathrm{m} / \mathrm{s})$ & $5.62 \pm 1.36$ & $4.09 \pm 1.11$ & $4.73 \pm 2.39$ \\
\hline
\end{tabular}

\begin{tabular}{c|c|c|c}
\hline C.elegans & Anterograde & Retrograde $\left(w_{1}\right)$ & Retrograde $\left(w_{2}\right)$ \\
\hline$\mu(\mu \mathrm{m} / \mathrm{s})$ & $1.79 \pm 0.04$ & $1.89 \pm 0.05$ & $5.11 \pm 0.40$ \\
\hline$\sigma(\mu \mathrm{m} / \mathrm{s})$ & $0.51 \pm 0.03$ & $0.67 \pm 0.03$ & $0.85 \pm 0.37$ \\
\hline$\xi$ & $-0.24 \pm 0.04$ & $-0.30 \pm 0.04$ & $-0.43 \pm 1.59$ \\
\hline$V_{\max }(\mu \mathrm{m} / \mathrm{s})$ & $3.98 \pm 0.30$ & $4.16 \pm 0.26$ & $5.90 \pm 7.88$ \\
\hline \hline Mammalian (Mouse) & Anterograde & Retrograde $\left(w_{1}\right)$ & Retrograde $\left(w_{2}\right)$ \\
\hline$\mu(\mu \mathrm{m} / \mathrm{s})$ & $\mathrm{xxx} \pm \mathrm{xxx}$ & $\mathrm{xxx} \pm \mathrm{xxx}$ & $\mathrm{xxx} \pm \mathrm{xxx}$ \\
\hline$\sigma(\mu \mathrm{m} / \mathrm{s})$ & $\mathrm{xxx} \pm \mathrm{xxx}$ & $\mathrm{xxx} \pm \mathrm{xxx}$ & $\mathrm{xxx} \pm \mathrm{xxx}$ \\
\hline$\xi$ & $\mathrm{xxx} \pm \mathrm{xxx}$ & $\mathrm{xxx} \pm \mathrm{xxx}$ & $\mathrm{xxx} \pm \mathrm{xxx}$ \\
\hline$V_{\max }(\mu \mathrm{m} / \mathrm{s})$ & $\mathrm{xxx} \pm \mathrm{xxx}$ & $\mathrm{xxx} \pm \mathrm{xxx}$ & $\mathrm{xxx} \pm \mathrm{xxx}$ \\
\hline
\end{tabular}

Table S1 Values of the fitting parameters $\mu, \sigma$ and $\xi$ of the Weibull distributions. The errors represent the fitting errors estimated by the maximum likelihood method (MLE) (top) and the statistical errors estimated by the bootstrapping method (bottom). 


\begin{tabular}{c|c}
\hline One-state model & Dynein \\
\hline$v_{\min }(\mu \mathrm{m} / \mathrm{s})$ & -0.1 \\
\hline$F_{\text {stall }}(\mathrm{pN})$ & 8 \\
\hline$k_{\mathrm{B}} \mathrm{T}(\mathrm{pN} \mathrm{nm})$ & $4.12(298 \mathrm{~K})$ \\
\hline$V_{\max }(\mu \mathrm{m} / \mathrm{s})$ & 20 \\
\hline
\end{tabular}

\begin{tabular}{c|c|c|}
\hline Three-state model & Kinesin & Dynein \\
\hline$\kappa_{1}(1 / \mathrm{s})$ & 110 & 203 \\
\hline$l(\mathrm{~nm})$ & \multicolumn{2}{|c|}{8.2} \\
\hline$\lambda_{1}(1000 / \mathrm{s})$ & 1.7 & 0.27 \\
\hline$d_{1}(\mathrm{~nm})$ & 3.5 & 2.1 \\
\hline$\lambda_{2}(1 / \mathrm{s})$ & 2.1 & 0.24 \\
\hline$d_{2}(\mathrm{~nm})$ & 0.27 & 1.8 \\
\hline$k_{\mathrm{B}} T(\mathrm{pN} \mathrm{nm})$ & \multicolumn{2}{|c}{$4.12(298 \mathrm{~K})$} \\
\hline$F_{\text {stall }}(\mathrm{pN})$ & \multicolumn{2}{|c}{} \\
\hline$V_{\text {max }}(\mu \mathrm{m} / \mathrm{s})$ & 4 & 20 \\
\hline
\end{tabular}

\begin{tabular}{c|c}
\hline $\begin{array}{c}\text { Energy-landscape } \\
\text { model }\end{array}$ & Kinesin \\
\hline$d(\mathrm{~nm})$ & 8 \\
\hline$[\mathrm{ATP}](\mathrm{mM})$ & 4 \\
\hline$k_{\text {cat }}^{0}(1 / \mathrm{s})$ & 488 \\
\hline$q_{\text {cat }}(1 / \mathrm{s})$ & 0.0062 \\
\hline$\delta(\mathrm{nm})$ & 3.7 \\
\hline$k_{b}^{0}(\mathrm{mM} / \mathrm{s})$ & 1300 \\
\hline$q_{\mathrm{b}}$ & 0.04 \\
\hline$k_{B} T(\mathrm{pN} \mathrm{nm})$ & $4.12(298 \mathrm{~K})$ \\
\hline$V_{\max }(\mu \mathrm{m} / \mathrm{s})$ & 4 \\
\hline
\end{tabular}

Table S2 Parameters used for chemo-mechanical models. 